\title{
Shuffling functors and spherical twists on $D^{\mathrm{b}}\left(\mathcal{O}_{0}\right)$
}

\author{
Fabian Lenzen, TU Munich
}

26th March 2021

\begin{abstract}
For a semisimple complex Lie algebra $\mathfrak{g}$, the BGG category $\mathcal{O}$ is of particular interest in representation theory. It is known that Irving's shuffling functors $\mathrm{Sh}_{w}$, indexed by elements $w \in W$ of the Weyl group, induce an action of the braid group $B_{W}$ associated to $W$ on the derived categories $D^{\mathrm{b}}\left(\mathcal{O}_{\lambda}\right)$ of blocks of $\mathcal{O}$.

We show that for maximal parabolic subalgebras $\mathfrak{p}$ of $\mathfrak{s l}_{n}$ corresponding to the parabolic subgroup $W_{\mathfrak{p}}=S_{n-1} \times S_{1}$ of $S_{n}$, the derived shuffling functors $\mathbf{L} \mathrm{Sh}_{s_{i}}$ are instances of Seidel and Thomas' spherical twist functors. Namely, we show that certain parabolic indecomposable projectives $P^{\mathfrak{p}}(w)$ are spherical objects, and the associated twist functors are naturally isomorphic to $\mathbf{L} \mathrm{Sh}_{w}[1]$ as auto-equivalences of $D^{\mathrm{b}}\left(\mathcal{O}^{\mathfrak{p}}\right)$.

We give an overview of the main properties of the BGG category $\mathcal{O}$, the construction of shuffling and spherical twist functors, and give some examples how to determine images of both. To this end, we employ the equivalence of blocks of $\mathcal{O}$ and the module categories of certain path algebras.
\end{abstract}

\section{Contents}

1 Introduction 2

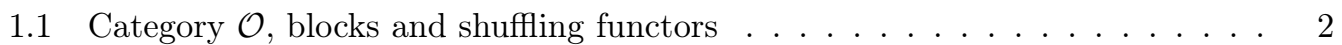

1.2 Actions of $W$ on $K_{0}\left(\mathcal{O}_{0}\right)$ and $D^{\mathrm{b}}\left(\mathcal{O}_{0}\right) \ldots \ldots \ldots \ldots$

1.3 Seidel and Thomas' spherical twist functors . . . . . . . . . . . . . . 4

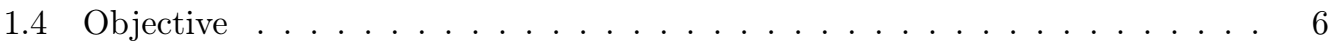

2 Some theory of the category $\mathcal{O}_{\lambda} \quad 7$

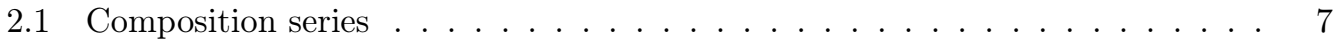

2.2 Kazhdan-Lusztig theory . . . . . . . . . . . . . . . . . . . . . . . . 8

2.3 Gradings . . . . . . . . . . . . . . . . . . . . . . . 8

2.4 Quivers . . . . . . . . . . . . . . . . . . . . . 10

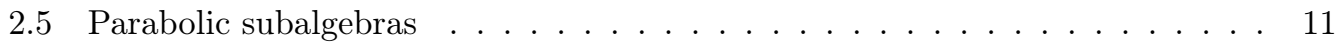

$3 B_{n}$-actions for $\mathfrak{s l}_{2} \quad \ldots 12$

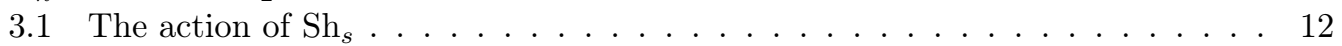

3.2 Spherical objects . . . . . . . . . . . . . . . . . . . . . . . . . . 13

3.3 Spherically twisting by $P(s) \ldots \ldots \ldots \ldots \ldots \ldots \ldots$

3.4 Spherically twisting by $L(e) \ldots \ldots \ldots \ldots \ldots$

$\begin{array}{lll}4 & B_{n} \text {-actions for } \mathfrak{s l}_{3} \text { and } \mathfrak{s l}_{n} & 19\end{array}$

4.1 Spherical subcategories . . . . . . . . . . . . . . . . . . . . . . . . 19

4.2 Maximal parabolic subalgebras . . . . . . . . . . . . . . 22

$5 \quad$ Further observations and final remarks 27

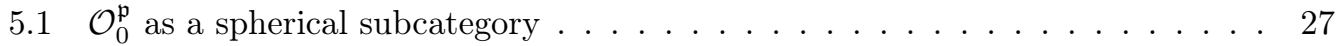

5.2 Necessity of extremal partitions . . . . . . . . . . . . . . . . 28

Published in Journal of Algebra, doi: 10.1016/j.jalgebra.2021.02.024. 
1 Introduction Consider a finite dimensional semisimple complex Lie algebra $\mathfrak{g}$ with Cartan subalgebra $\mathfrak{h}$ that gives rise to a root system $\Phi$ and a root space decomposition $\mathfrak{g}=\bigoplus_{\alpha \in \Phi} \mathfrak{g}_{\alpha}$. A choice of simple roots $\Delta$ fixes the positive roots $\Phi^{+}$, the corresponding subalgebra $\mathfrak{n}:=\bigoplus_{\alpha \in \Phi^{+}} \mathfrak{g}_{\alpha}$ and the corresponding Borel subalgebra $\mathfrak{b}=\mathfrak{h} \oplus \mathfrak{n}$.

Representations of $\mathfrak{g}$ are equivalent to modules over the universal enveloping algebra $U(\mathfrak{g})$ [Hum72, $\S \mathrm{V}]$. The $B G G$ category $\mathcal{O}$ of $\mathfrak{g}$ is the full subcategory of $U(\mathfrak{g})$-Mod consisting of modules that $(\mathcal{O} 1)$ are finitely generated, $(\mathcal{O} 2)$ have a weight space decomposition $M=\bigoplus_{\lambda \in \mathfrak{h}^{*}} M_{\lambda}$ and (O3) are locally $\mathfrak{n}$-finite; i. e., for every $v \in M$, the orbit $U\left(\mathfrak{n}^{+}\right) v$ is finite dimensional.

1.1 Category $\mathcal{O}$, blocks and shuffling functors Denote the Weyl group of $\mathfrak{g}$ by $W$. The half-sum of positive roots $\rho:=\frac{1}{2} \sum_{\alpha \in \Phi^{+}} \alpha$ gives rise to the dot-action $w \cdot \lambda \mapsto w(\lambda+\rho)-\rho$. The C-span of $\Phi$ is a Euclidean space and its inner product $(-,-)$ fixes the coroots $\check{\alpha}:=\frac{2 \alpha}{(\alpha, \alpha)}$ and the fundamental weights $\varpi_{\alpha}$ for $\alpha \in \Delta$ defined by $\frac{\left(\varpi_{\alpha}, \beta\right)}{(\beta, \beta)}$ for $\alpha, \beta \in \Delta$. We call a weight $\lambda \in \mathbf{C} \Phi$ integral if $\frac{2(\lambda, \alpha)}{(\alpha, \alpha)} \in \mathbf{Z}$ and denote by $\Lambda$ the set of all integral weights. We call a $\mathbf{Z}_{\geq 0}$-linear combination of the $\varpi_{\alpha}$ 's dominant and denote the set of all dominant weights by $\Lambda^{+}$. We call a weight $\lambda \rho$-dominant if $\frac{2(\lambda+\rho, \alpha)}{(\alpha, \alpha)} \notin \mathbf{Z}_{<0}$ for all $\alpha \in \Delta$. To a weight $\lambda$ we associate the subgroup $W_{\lambda}:=\left\langle s_{\alpha} \mid \frac{2(\lambda+\rho, \alpha)}{(\alpha, \alpha)} \in \mathbf{Z}\right\rangle \leq W$; See [Hum08; Jan79] for details.

The category $\mathcal{O}$ has a decomposition $\mathcal{O}=\bigoplus_{\lambda} \mathcal{O}_{\lambda}$ into blocks $\mathcal{O}_{\lambda}$, indexed by the $\rho$ dominant weights $\lambda$, each of which consists of the modules of highest weight in $W_{\lambda} \cdot \lambda$ $[\text { Hum08, thm 4.9 }]^{1}$. In particular, Each block $\mathcal{O}_{\lambda}$ contains the simple modules $L(w \cdot \lambda)$, the indecomposable projectives $P(w \cdot \lambda)$ and the Verma modules $M(w \cdot \lambda)$ of highest weight $w \in W / W_{\lambda}$. If a block $\mathcal{O}_{\lambda}$ is fixed, we just write $L(w), P(w)$ and $M(w)$ for the respective objects therein. Each block $\mathcal{O}_{\lambda}$ is Morita equivalent to modules over a quasi-hereditary algebra [BGG76].

A weight $\lambda$ is called regular if its stabilizer subgroup $W_{\lambda}$ w.r.t. the dot-action is trivial; i. e., if $\lambda$ does not lie on any reflection plane. All blocks $\mathcal{O}_{\lambda}$ associated to regular weights are equivalent as categories; in the following we shall thus work in the block $\mathcal{O}_{0}$ containing the trivial $\mathfrak{g}$-representation $L(e \cdot 0)=\mathbf{C}$, which is called the principal block.

Definition 1.1. A Coxeter system consists of a group $W$, a fixed set $S$ of generators and a presentation $W=\langle s \in S| s^{2}=e$, sts $\left.\cdots=t s t \cdots\right\rangle$ with $m_{s t}$ factors $s, t$ on both sides. The $s \in S$ are called simple reflections. The matrix $\left(m_{s t}\right)_{s, t \in W}$ is called the Coxeter matrix of $W$. To $W$, there is the associated braid group $B_{W}=\langle s \in S|$ sts $\cdots=$ tst $\left.\cdots\right\rangle$, such that there is a natural quotient map $B_{W} \rightarrow W$. A finite Coxeter system has a length function $\ell: W \rightarrow \mathbf{N}_{0}$, which assigns to an element $w \in W$ the length of a shortest expression for $w$ in terms of $S$. With respect to $\ell$, there is a unique longest element $w_{0} \in W$.

Example 1.2. The Weyl group of $\mathfrak{g}$ is a Coxeter group. In particular, the symmetric group $S_{n}$, which is the Weyl group of $\mathfrak{s l}_{n}$, is a Coxeter group, generated by the simple reflections $s_{1}, \ldots, s_{n-1}$. Its Coxter matrix has entries $m_{s_{i}, s_{j}}=\left\{\begin{array}{l}1 \text { if } i=j \\ 3 \text { if }|i-j|=1 . \text { For the symmetric group, } \\ 2 \text { otherwise }\end{array}\right.$ $B_{n}:=B_{S_{n}}$ is the well-known Artin braid group.

For weights $\lambda, \mu \in \mathfrak{h}^{*}$ with $\lambda-\mu \in \Lambda$, there is a unique $\nu \in W(\mu-\lambda)$ (w.r.t. the ordinary $W$-action) such that the simple module $L(\nu)$ is finite-dimensional, which is the case if and only if $\nu$ is dominant [Hum08, thm. 1.6].

\footnotetext{
${ }^{1}$ Humphreys indexes blocks by $\rho$-antidominant weights, which results in slightly different formulation for some statements.
} 
Definition 1.3 [Jan79, p. 54]. The translation functor $T_{\lambda}^{\mu}: \mathcal{O}_{\lambda} \rightarrow \mathcal{O}_{\mu}$ assigns to $M$ the direct summand of $M \otimes L(\nu)$ lying in $\mathcal{O}_{\mu}$.

The functor $T_{\lambda}^{\mu}$ is exact, preserves projectives, commutes with duality, and is biadjoint to $T_{\mu}^{\lambda}$. From now on, we assume that $\lambda, \mu$ are integral. For most of what we need, this is stricter than necessary, but sufficient for our needs; see [Hum08, §7] for a more general treatment and for an overview of properties of $T_{\lambda}^{\mu}$.

Definition 1.4. Let $s \in W$ be a simple reflection and $\mu$ be an integral weight with stabiliser $W_{\mu}=\{e, s\}$. The translation through the $s$-wall is the composition $\Theta_{s}:=T_{\mu}^{0} T_{0}^{\mu}: \mathcal{O}_{0} \rightarrow \mathcal{O}_{0}$.

As notation suggests, $\Theta_{s}$ is independent of the choice of $\mu$. It is an exact self-adjoint auto-equivalence of the block $\mathcal{O}_{0}[\mathrm{Jan} 79, \S 2.10]$. It is uniquely determined by the existence of short exact sequences

$$
0 \rightarrow M(w) \rightarrow \Theta_{s} M(w) \rightarrow M(w s) \rightarrow 0 \quad \text { and } \quad \Theta_{s} M(w) \cong \Theta_{s} M(w s)
$$

for $w<w s$ [Jan79, Satz 2.10]. Furthermore, $\Theta_{s}^{2}=\Theta_{s} \oplus \Theta_{s}$ [Hum08, §7.14].

Definition 1.5 [Car86, §2; Irv93, §3]. From the adjunctions $T_{\mu}^{0} \dashv T_{0}^{\mu}$ and $T_{0}^{\mu} \dashv T_{\mu}^{0}$ we get adjunction maps $\eta_{s}$ : id $\Rightarrow \Theta_{s}$ and $\varepsilon_{s}: \Theta_{s} \Rightarrow$ id, and we define the mutually adjoint shuffling and the coshuffling functor $\mathrm{Sh}_{s}$ and $\mathrm{Csh}_{s}$ as

$$
\mathrm{Sh}_{s}:=\operatorname{coker}\left(\eta_{s}\right): \mathcal{O}_{0} \rightarrow \mathcal{O}_{0}, \quad \operatorname{Csh}_{s}:=\operatorname{ker}\left(\varepsilon_{s}\right): \mathcal{O}_{0} \rightarrow \mathcal{O}_{0} .
$$

In particular, (1.1) implies that for $w<w s$,

$$
\mathrm{Sh}_{s} M(w)=M(w s) \quad \text { and } \quad \operatorname{Csh}_{s} M(w s)=M(w) .
$$

By the snake lemma, $\mathrm{Sh}_{s}$ is right exact and $\mathrm{Csh}_{s}$ is left exact, so we can consider the mutually inverse [MS05, thm. 5.7] derived functors $\mathbf{L} \mathrm{Sh}_{s}$ and $\mathbf{R} \mathbf{C s h}_{s}$.

In general, we can see an adjunction morphism $\eta_{s}$ on an abelian category $\mathcal{C}$ as a functor from $\mathcal{C}$ to the arrow category $\mathcal{C}^{[1]}$. By the snake lemma, the cokernel is a right exact functor functor $\mathcal{C}^{[1]} \rightarrow \mathcal{C}$, whose derived functor $\mathbf{L}$ coker: $D^{\mathrm{b}}\left(\mathcal{C}^{[1]}\right) \rightarrow D^{\mathrm{b}}(\mathcal{C})$ is the mapping cone [Gro16, thm 3.5.6]. ${ }^{2}$ We thus obtain that $\mathbf{L} \mathrm{Sh}_{s} \cong \operatorname{cone}\left(\eta_{s}\right)$ and dually that $\mathbf{R} \mathbf{C s h}_{s} \cong$ $\operatorname{cocone}\left(\varepsilon_{s}\right)=\operatorname{cone}\left(\varepsilon_{s}\right)[1]$. The snake lemma also implies that $\mathbf{L}^{i} \mathrm{Sh}_{s}=0$ for $i \neq 0,1$ and $\mathbf{R}_{j} \operatorname{Csh}_{s}=0$ for $j \neq-1,0$.

Definition 1.6. We say that an object $M \in \mathcal{C}$ is $F$-acyclic for a left or right exact functor $F: \mathcal{C} \rightarrow \mathcal{D}$ of abelian categories if $\mathbf{R}_{i} F M=0$ or $\mathbf{L}^{i} M=0$ for $i \neq 0$, respectively.

Projective (respectively, injective) objects are acyclic for every right (resp., left) exact functor. Since the adjunction unit $\eta_{s}: M(w) \rightarrow \Theta_{s}$ is injective for all $w \in W$ [Hum08, thm 12.2], every $M(w)$ is both $\mathrm{Sh}_{s}$-acyclic.

\footnotetext{
${ }^{2}$ As the inclusion $D^{\mathrm{b}}\left(\mathcal{C}^{[1]}\right) \hookrightarrow D^{\mathrm{b}}(\mathcal{C})^{[1]}$ is no equivalence in general, one has to pay attention to the fact that the mapping cone is a functor on coherent diagrams $D^{\mathrm{b}}\left(\mathcal{C}^{[1]}\right)$, but not necessarily on general diagrams $D^{\mathrm{b}}(\mathcal{C})^{[1]}$.
} 


\subsection{Actions of $W$ on $K_{0}\left(\mathcal{O}_{0}\right)$ and $D^{\mathrm{b}}\left(\mathcal{O}_{0}\right)$}

Definition 1.7. The Grothendieck group $K_{0}(\mathcal{C})$ of an abelian category $\mathcal{C}$ is the abelian group generated by symbols $[M]$ for the objects $M \in \mathcal{C}$, subject to the relation $[E]=[A]+[B]$ whenever $E$ is an extension of $A$ by $B$. Similarly, the Grothendieck group $K_{0}(\mathcal{T})$ of a triangulated category $\mathcal{T}$ is the abelian group generated by symbols $[M]$ for the objects $M \in \mathcal{T}$, subject to the relation $[E]=[A]+[B]$ whenever $A \rightarrow E \rightarrow B \rightarrow A[-1]$ is a triangle.

An exact (respectively, triangulated) functor induces a group homomorphism on $K_{0}(\mathcal{C})$ (respectively, $K_{0}(\mathcal{T})$ ). The inclusion $\mathcal{C} \hookrightarrow D^{\mathrm{b}}(\mathcal{C})$ induces an isomorphism $K_{0}(\mathcal{C}) \cong K_{0}\left(D^{\mathrm{b}}(\mathcal{C})\right)$ of abelian groups [Gro57].

For the category $\mathcal{O}_{0}$, the group $K_{0}\left(\mathcal{O}_{0}\right)$ is free, and each of the collections $\{L(w)\}_{w \in W}$, $\{M(w)\}_{w \in W}$ and $\{P(w)\}_{w \in W}$ is a $\mathbf{Z}$-basis of it. For the simple modules, this follows from the fact that every object an $\mathcal{O}_{0}$ has a composition series (see section 2.1), and for the others from the fact that each $[L(w)]$ can be written uniquely in terms of the $M(u)$ 's or $P(v)$ 's, due to the composition series of the latter.

The shuffling functors induce a right action of $W$ on $K_{0}\left(\mathcal{O}_{0}\right)$, given for a $w \in W$ and a simple reflection $s \in W$ by the assignment

$$
W \longrightarrow \operatorname{Aut}\left(K_{0}\left(\mathcal{O}_{0}\right)\right), \quad s \longmapsto\left[\mathrm{Sh}_{s}\right]:[M(w)] \mapsto[M(w s)] ;
$$

indeed, we even have $\mathrm{Sh}_{s} M(w)=M(w s)$ for $w \leq w s$ and from (1.1) and from the $\mathrm{Sh}_{s^{-}}$ acyclicity of Verma modules we learn that $M(w)$ and $\mathrm{Sh}_{s}(w s)$ have the same composition factors such that $[M(w)]=\sum_{v}[M(w): L(v)][L(v)]=[M(w s)]$; hence, the above action is well-defined on $W$. This action actually comes from an action on the derived category $\mathcal{O}_{0}$ :

Theorem 1.8 [Rou06, thm. 10.4]. The assignment

$$
B_{W} \rightarrow \operatorname{Aut}\left(D^{\mathrm{b}}\left(\mathcal{O}_{0}\right)\right), \quad s \mapsto \mathbf{L} \mathrm{Sh}_{s}, \quad s^{-1} \mapsto \mathbf{R} \mathrm{Csh}_{s}
$$

defines a weak action of $B_{W}$ on the derived category $D^{\mathrm{b}}\left(\mathcal{O}_{0}\right)$, meaning that for simple reflections $s, t \in W$, there are natural isomorphisms $\mathbf{L} \mathrm{Sh}_{s} \mathbf{L} \mathrm{Sh}_{t} \cong \mathbf{L} \mathrm{Sh}_{t} \mathbf{L} \mathrm{Sh}_{s}$ whenever $s t=t s$ and $\mathbf{L} \mathrm{Sh}_{s} \mathbf{L} \mathrm{Sh}_{t} \mathbf{L} \mathrm{Sh}_{s} \cong \mathbf{L} \mathrm{Sh}_{t} \mathbf{L} \mathrm{Sh}_{s} \mathbf{L} \mathrm{Sh}_{t}$ whenever sts $=t s t$, and the following square commutes:

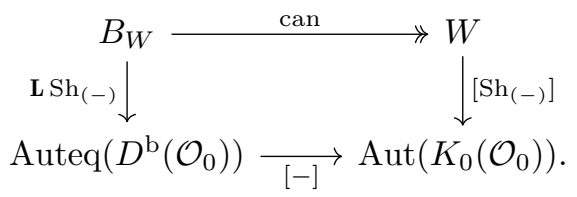

1.3 Seidel and Thomas' spherical twist functors Let $k$ be a field and $\mathcal{C}$ be a $k$-linear abelian category $\mathcal{C}$ of finite global dimension. Seidel and Thomas have constructed an action of the braid group $B_{n}:=B_{S_{n}}$ of the symmetric group $S_{n}$ on $\mathcal{C}$ in terms of spherical objects [ST01]. We give a short summary of their construction.

Definition 1.9. Let $X, Y \in \operatorname{Ch}(\mathcal{C})$ be chain complexes. We employ the convention that the homological shift upwards; i. e., $X[1]_{j}:=X_{j-i}$. We define the graded $k$-vector space $\operatorname{Hom}_{D^{\mathrm{b}}(\mathcal{C})}^{*}(X, Y)$ with graded components $\operatorname{Hom}_{D^{\mathrm{b}}(\mathcal{C})}^{j}(X, Y):=\operatorname{Hom}_{D^{\mathrm{b}}(\mathcal{C})}(X[j], Y)$.

We denote by $\operatorname{hom}_{\mathcal{C}}^{\bullet}(X, Y) \in \mathrm{Ch}(k$-Vect $)$ the chain complex with homological components $\operatorname{hom}_{\mathcal{C}}^{j}(X, Y):=\operatorname{Hom}_{k}(X[j], Y)$ the graded $k$-linear morphisms $X \rightarrow Y$ (not necessarily chain maps), and differential $\mathrm{d}_{\text {hom }}$ defined on homogeneous elements of degree $j$ by $\mathrm{d}_{\text {hom }}(f)=$ $\mathrm{d}_{Y} f-(-1)^{j} f \mathrm{~d}_{X}$. Its cohomology $H^{j} \operatorname{hom}_{\mathcal{C}}^{\bullet}(X, Y)=\operatorname{Hom}_{K^{\mathrm{b}}(\mathcal{C})}(Y[j], Y)$ is the (honest) the Hom-space in the bounded homotopy category $K^{\mathrm{b}}(\mathcal{C})$ [Wei94, §2.7.5]. 
Definition 1.10. Let $X \in \mathrm{Ch}(\mathcal{C})$ and $V \in \operatorname{Ch}(k$-Vect). Since $\mathcal{C}$ is a $k$-linear category, we can regard $X$ as a complex of vector spaces, on which a $\lambda \in k$ acts by $\lambda x:=\left(\lambda \operatorname{id}_{X}\right)(x)$ for $x \in X$. We define the complex $\operatorname{lin}_{\mathbf{C}}^{\bullet}(V, X) \in \mathrm{Ch}(\mathcal{C})$ of $k$-linear maps from $V$ to $X$ with differential $\left(\mathrm{d}_{\operatorname{lin}_{\mathrm{C}}} f\right) v:=(-1)^{\operatorname{deg} v}[\mathrm{~d} f v-f \mathrm{~d} v]$ and the tensor product complex $V \otimes X$ with differential $\mathrm{d}_{V \otimes X}(v \otimes x):=\mathrm{d} v \otimes x+(-1)^{\operatorname{deg} v} v \otimes \mathrm{d} x$ for homogeneous $v$.

Remark 1.11. For a homogeneous basis $B$ of $V$ and $v \in V$, let $\beta_{b}(v)$ be the coefficients such that $v=\sum_{b \in B} \beta_{b}(v) b$. There are homogeneous isomorphisms of graded vector spaces

$$
\begin{array}{rlrl}
V \otimes X & \stackrel{\cong}{\longmapsto} \bigoplus_{b \in B} X[-\operatorname{deg} b]_{b}, & \operatorname{lin}_{\mathbf{C}}^{\bullet}(V, X) & \stackrel{\cong}{\longmapsto} \prod_{b \in B} X[\operatorname{deg} b]_{b}, \\
v \otimes x & \longmapsto \sum_{b} \beta_{b}(v) x_{b} & f \longmapsto(f(b))_{b} ;
\end{array}
$$

The differential on $V \otimes X$ and $\operatorname{lin}_{\mathbf{C}}^{\bullet}(V, X)$ is specified by

$$
\begin{aligned}
\mathrm{d}_{V \otimes X}\left(x_{b}\right) & =\sum_{b^{\prime} \in B} \beta_{b^{\prime}}(\mathrm{d} b) x_{b^{\prime}}+(-1)^{\operatorname{deg} b}(\mathrm{~d} x)_{b}, \\
\mathrm{~d}_{\operatorname{lin}_{\mathbf{C}}^{\bullet}(V, X)}\left(\left(x_{b}\right)_{b \in B}\right) & =\left((-1)^{\operatorname{deg} b}\left(\mathrm{~d} x_{b}-\left(\sum_{b^{\prime} \in B} \beta_{b}\left(\mathrm{~d} b^{\prime}\right) x_{b}\right)\right)\right)_{b \in B} ;
\end{aligned}
$$

if $V$ is degree-wise finite dimensional, the complex $\operatorname{lin}_{\mathbf{C}}(V, X) \cong V^{*} \otimes X$ is spanned by maps $x_{b}: b^{\prime} \mapsto \delta_{b b^{\prime}} x$ (where $\delta$ denotes the Kronecker- $\delta$ ), and we get

$$
\mathrm{d}_{\operatorname{lin}_{\dot{\mathrm{C}}}(V, X)}\left(x_{b}\right)=(-1)^{\operatorname{deg} b}\left(\left(\mathrm{~d} x_{b}\right)-\sum_{b^{\prime} \in B} \beta_{b}\left(\mathrm{~d} b^{\prime}\right) x_{b^{\prime}}\right) .
$$

Definition 1.12. An object $E \in D^{\mathrm{b}}(\mathcal{C})$ is called $d$-spherelike for $d \geq 0$ if

$(\mathrm{S} 1)$ for any $F \in D^{\mathrm{b}}(\mathcal{C})$, the spaces $\operatorname{Hom}_{D^{\mathrm{b}}(\mathcal{C})}^{*}(E, F)$ and $\operatorname{Hom}_{D^{\mathrm{b}}(\mathcal{C})}^{*}(F, E)$ are of finite total dimension, and

$(\mathrm{S} 2) \operatorname{Hom}_{D^{\mathrm{b}}(\mathcal{C})}^{*}(E, E) \cong k[x] /\left(x^{2}\right)$ as graded algebras, where $x$ is a morphism of degree $d$.

It is called $d$-spherical if it also has the Calabi-Yau-property:

(S3) For all $F$ and $i$, composition of morphisms gives a non-degenerate pairing

$$
\operatorname{Hom}_{D^{\mathrm{b}}}^{i}(F, E) \otimes \operatorname{Hom}_{D^{\mathrm{b}}}^{d-i}(E, F) \rightarrow \operatorname{Hom}_{D^{\mathrm{b}}}^{d}(E, E) \rightarrow k x .^{3}
$$

Definition 1.13. For $\Xi_{E} F:=\operatorname{hom}_{\mathcal{C}}(E, F) \otimes E$ and $\Xi_{E}^{\prime} F:=\operatorname{lin}_{\mathbf{C}}\left(\operatorname{hom}_{\mathcal{C}}^{\bullet}(F, E), E\right)$, we consider the evaluation and coevaluation maps

$$
e v: \Xi_{E} F \rightarrow F, \phi \otimes f \mapsto \phi(f) ; \quad \quad e v^{\prime}: F \rightarrow \Xi_{E}^{\prime} F, f \mapsto \phi \mapsto \phi(f) .
$$

If $E$ is $d$-spherelike, we define its spherical twist and cotwist functor

$$
T_{E}:=\operatorname{cone}\left(e v: \Xi_{E} \Rightarrow \operatorname{id}_{\mathcal{C}}\right), \quad \quad T_{E}^{\prime}:=\operatorname{cocone}\left(e v^{\prime}: \operatorname{id}_{\mathcal{C}} \Rightarrow \Xi_{E}^{\prime}\right) ;
$$

here, the grading of the $(c o)$ cone is such that $\mathrm{id}_{\mathcal{C}}$ is in degree zero.

Isomorphic objects in $D^{\mathrm{b}}(\mathcal{C})$ give rise to naturally isomorphic twist and cotwist functors [ST01, prop. 2.6], hence $T_{E}$ and $T_{E}^{\prime}$ are well-defined. There is an adjunction $T_{E}^{\prime} \dashv T_{E}$ [ST01, lem. 2.8].

\footnotetext{
${ }^{3}$ If $d \neq 0$, we already have $\operatorname{Hom}_{D^{\mathrm{b}}}^{d}(E, E)=k x$, but if $d=0$, we have $\operatorname{Hom}_{D^{\mathrm{b}}}^{d}(E, E)=k x \oplus k \mathrm{id}_{E}$. In this case, we mean that o: $\operatorname{Hom}_{D^{\mathrm{b}}}^{i}(F, E) \otimes \operatorname{Hom}_{D^{\mathrm{b}}}^{d-i}(F, E) \rightarrow \operatorname{Hom}_{D^{\mathrm{b}}}^{d}(E, E) / k \mathrm{id}_{E} \cong k$ be non-degenerate.
} 
Remark 1.14. In terms of the descriptions of $\operatorname{lin}_{\mathbf{C}}^{\bullet}$ and $\otimes$ w.r.t. fixed bases $A$ and $B$ of $\operatorname{hom}_{\mathcal{C}}(E, F)$ and $\operatorname{hom}_{\mathcal{C}}^{\bullet}(F, E)$ from remark 1.11, the evaluation and coevaluation map are the homogeneous maps

$$
\begin{array}{rlrl}
e v: \Xi_{E} F=\bigoplus_{a \in A} E[\operatorname{deg} a]_{a} & \longrightarrow F & e v^{\prime}: F & \longrightarrow \prod_{b \in B} F[-\operatorname{deg} b]_{b}=\Xi_{E}^{\prime} F \\
\left(e_{a}\right)_{a} & \longmapsto \sum_{a} a\left(e_{a}\right) & v & \longmapsto(b(v))_{b} .
\end{array}
$$

Similarly, a morphism $f: F \rightarrow F^{\prime}$ of complexes induces maps

$$
\begin{aligned}
f_{*}: \Xi_{E} F & \longrightarrow \Xi_{E} F^{\prime} & f_{*}: \Xi_{E}^{\prime} F & \longrightarrow \Xi_{E}^{\prime} F^{\prime} \\
g \otimes e & \longmapsto f g \otimes e & \longmapsto h & \mapsto \psi(h f),
\end{aligned}
$$

which correspond to

$$
\begin{array}{rlrl}
\bigoplus_{a \in A} E[\operatorname{deg} a] & \longrightarrow \bigoplus_{a^{\prime} \in A^{\prime}} E\left[\operatorname{deg} a^{\prime}\right] & \prod_{b \in B} E[-\operatorname{deg} a] & \longrightarrow \prod_{b^{\prime} \in B^{\prime}} E\left[-\operatorname{deg} b^{\prime}\right] \\
e_{a} & \longmapsto \sum_{a^{\prime} \in A^{\prime}}\left(\beta_{a^{\prime}}(a f) e_{a}\right)_{a^{\prime}} & \left(e_{b}\right)_{b} \longmapsto\left(\sum_{b \in B} \beta_{b}\left(b^{\prime} f\right) e_{b}\right)_{b^{\prime}} .
\end{array}
$$

Proposition 1.15 [ST01, prop. 2.10]. If $E$ is $d$-spherical, then $T_{E}$ and $T_{E}^{\prime}$ are mutually inverse auto-equivalences of $D^{\mathrm{b}}(\mathcal{C})$.

Notation 1.16. Given a double (or triple) complex $X^{\bullet \bullet}$, we denote its $\oplus$-total complex by $\left\{X^{\bullet \bullet}\right\}$. In particular, since we can regard a morphism $f: X \rightarrow Y$ of chain complexes trivially as a double complex with $Y$ placed in degree zero, the mapping cone can be written as cone $(f)=\{f: X \rightarrow Y\}$. We occasionally write a zero below the complex placed in degree zero, so cone $(f)=\{f: X \rightarrow \underset{0}{Y}\}$.

Remark 1.17. Since for every $d$-spherical object $E$, $\operatorname{hom}_{\mathcal{C}}^{\bullet}(E, E) \cong\langle\mathrm{id}\rangle_{k} \oplus\langle x\rangle_{k}[d]$ as complexes of vector spaces, we have

$$
T_{E}^{\prime} E=\left\{\left(\begin{array}{l}
1 \\
x
\end{array}\right): \underset{0}{E} \rightarrow E \oplus E[-d]\right\} \simeq E[1-d] .
$$

Definition 1.18. A collection $\left\{E_{1}, \ldots, E_{n}\right\}$ of $d$-spherical objects is an $\mathrm{A}_{n}$-configuration if $\operatorname{dim} \operatorname{Hom}_{D^{\mathrm{b}}(\mathcal{C})}^{*}\left(E_{i}, E_{j}\right)=\left\{\begin{array}{l}1 \text { if }|i-j|=1, \\ 0 \text { otherwise }\end{array}\right.$.

Theorem 1.19 [ST01, thm. 2.17]. Given an $\mathrm{A}_{n}$-configuration $\left\{E_{1}, \ldots, E_{n}\right\}$ of $d$-spherical objects, the assignment

$$
B_{n} \rightarrow \operatorname{Auteq}\left(D^{\mathrm{b}}(\mathcal{C})\right), \quad s_{i} \mapsto T_{E_{i}}
$$

defines a weak action of the braid group.

1.4 Objective The category $\mathcal{O}_{0}$ is a $\mathbf{C}$-linear category of finite global dimension and hence satisfies the requirements of [ST01]. For $\mathfrak{g}=\mathfrak{s l}_{n}$, we want to understand whether there is an $\mathrm{A}_{n}$-configuration in $D^{\mathrm{b}}\left(\mathcal{O}_{0}\right)$ such that the associated twist functors relate to the shuffling functors.

We shall show in theorem 3.6 that for $\mathfrak{g}=\mathfrak{s l}_{2}$, derived shuffling functors are in instance of spherical twists for suitable objects, and we shall develop the following generalisation.

For a parabolic subalgebra $\mathfrak{p} \subseteq \mathfrak{s l}_{n}$ corresponding to a parabolic subgroup $W_{\mathfrak{p}} \leq S_{n}$, we write $\mathcal{O}_{0}^{\mathfrak{p}}$ for the principal block of the parabolic category $\mathcal{O}$, see section 2.5. We shall prove the following: 
Theorem 4.14. For a maximal parabolic subalgebra $\mathfrak{p}$ of $\mathfrak{s l}_{n}$ corresponding to the parabolic subgroup $S_{n-1} \times S_{1} \leq S_{n}$, the derived category $D^{\mathrm{b}}\left(\mathcal{O}_{0}^{\mathfrak{p}}\right)$ contains an $\mathrm{A}_{n-1}$-configuration of 0 -spherical objects such that the associated spherical twist functors and the restriction of $\mathbf{L} \mathrm{Sh}_{s_{i}}[1]$ to $D^{\mathrm{b}}\left(\mathcal{O}_{0}^{\mathfrak{p}}\right)$ are naturally isomorphic auto-equivalences of $D^{\mathrm{b}}\left(\mathcal{O}_{0}^{\mathfrak{p}}\right)$.

Outline Section 2 gives an overview of some of the most important properties of blocks of $\mathcal{O}$ and the tools we employ. We do not require any prior knowledge about $\mathcal{O}$. We include a short refresher on Kazhdan-Lusztig theory, quivers and graded algebras.

In Section 3, we explain how to compute images of the shuffling functors for the special case $\mathfrak{g}=\mathfrak{s l}_{2}$ explicitly. The proof of theorem 3.6 will serve as a model for the proof of the more general theorem 4.14 , which is worked out in section 4 .

2 Some theory of the category $\mathcal{O}_{\lambda}$ In this section, we collect the most important properties of $\mathcal{O}_{\lambda}$. For simplicity, we always assume that $\lambda$ is an integral $\rho$-dominant weight.

2.1 Composition series Every module $M \in \mathcal{O}_{\lambda}$ admits a composition series

$$
0 \subsetneq M_{1} \subsetneq \cdots \subsetneq M_{\ell}=M
$$

with subquotients $M_{i} / M_{i-1}$ isomorphic to the simple modules $L(w \cdot \lambda)$ [Jan79, Satz 1.13]. According to the Jordan-Hölder theorem, any composition series for $M$ involves the same isomorphism classes of the simple factors, up to their order of appearance. The multiplicity of $L(w \cdot \lambda)$ in any composition series for $M$ is denoted by $[M: L(w \cdot \lambda)]$. There is another important filtration:

Definition 2.1. A Loewy filtration of a module $M \in \mathcal{O}_{0}$ is a filtration of minimal length with semisimple subquotients $M_{i}$. A module $M$ is called rigid if it has a unique Loewy filtration; for instance, Verma modules are rigid [Irv88, thm. 1]. The socle $\operatorname{soc} M$ and the head hd $M$ of a module $M$ are its maximal semisimple submodule and quotient, respectively.

Notation 2.2. We write Loewy filtrations diagrammatically by putting all simple summands of each semisimple subquotient into a common horizontal layer, from the socle in the bottom line to the head in the top line.

Example 2.3. For $\mathfrak{g}=\mathfrak{s l}_{2}$, the non-simple Verma module has a composition series (and also Loewy filtration) $M(e)=\left(\begin{array}{l}L(e) \\ L(s)\end{array}\right)$, which is just another way to say that there is a short exact sequence $0 \rightarrow L(s) \rightarrow M(e) \rightarrow L(e) \rightarrow 0$.

For $\mathfrak{g}=\mathfrak{s l}_{3}$, the Verma module $M(s)=\left(\begin{array}{c}L(s) \\ L(s t) \\ L\left(w_{0}\right)\end{array}\right)$ has the simple quotient $L(s)$ and the simple submodule $L\left(w_{0}\right)$. Its non-trivial submodules have Loewy filtrations $L\left(w_{0}\right)$, $\left(\begin{array}{c}L(s t) \\ L\left(w_{0}\right)\end{array}\right),\left(\begin{array}{c}L(t s) \\ L\left(w_{0}\right)\end{array}\right)$ and $\left(\begin{array}{cc}L(s t) & L(t s) \\ L\left(w_{0}\right)\end{array}\right)$.

Remark 2.4. For a morphism $f: M \rightarrow N$ in $\mathcal{O}$ there exist composition series of $M$ and $N$ contiguously containing a composition series of $\operatorname{im} f$ at the top and the bottom, respectively. The cokernel and kernel of $f$ comprise the composition factors of $M$ and $N$ not belonging to $\operatorname{im} f$, respectively. For example, for $\mathfrak{g}=\mathfrak{s l}_{2}$, we can write the short exact sequence $0 \rightarrow M(e) \rightarrow P(s) \rightarrow M(s) \rightarrow 0$ diagrammatically as

$$
\begin{aligned}
& L(e) \\
& \underbrace{L(s)}_{M(e)} \hookrightarrow \underbrace{L(s)}_{P(s)} \begin{array}{l}
L(e) \\
L(s)
\end{array} \rightarrow \underbrace{L(s)}_{M(s) .} \\
&
\end{aligned}
$$


Since $\operatorname{dim} \operatorname{Hom}_{\mathcal{O}}(L(v), L(w))=\delta_{v w}$, a morphism is determined by a scalar for each pair of composition factors from its domain and its codomain.

Certain modules, such as the indecomposable projectives $P(w \cdot \lambda)$, also admit a standard filtration whose subquotients are isomorphic to Verma modules $M(v \cdot \lambda)$. We write $(P(w \cdot \lambda)$ : $M(v \cdot \lambda)$ ) for the (unique) multiplicity of $M(v \cdot \lambda)$ in a standard filtration of $P(w \cdot \lambda)$.

Theorem $2.5(B G G$ reciprocity) [BGG76]. $(P(v \cdot \lambda): M(w \cdot \lambda))=[M(w \cdot \lambda): L(v \cdot \lambda)]$.

2.2 Kazhdan-Lusztig theory There is a $W^{2}$-parametrised collection $\left\{p_{v w}\right\}$ of polynomials in $\mathbf{Z}\left[q^{ \pm 1}\right]$, called Kazhdan-Lusztig polynomials, which occur as base change coefficients between the standard basis and the Kazhdan-Lusztig basis (usually denoted, respectively, by $\left\{T_{w}\right\}$ and $\left\{C_{w}\right\}$ for $w \in W$ ) of the Iwahori-Hecke algebra $\mathrm{H}_{q}(W)$ [KL79; Soe97], which is a $\mathbf{Z}\left[q^{ \pm 1 / 2}\right]$-algebra and a deformation of the group algebra $\mathbf{Z W}$. The following relation to composition factor multiplicities has been conjectured in [KL79, conjecture 1.5]:

Theorem 2.6 (Kazhdan-Lusztig conjecture) [BB81, §4; BK81, §8]. The composition factor multiplicities in a block $\mathcal{O}_{\lambda}$ for a regular weight $\lambda$ are given by

$$
(P(v \cdot \lambda): M(w \cdot \lambda))=[M(w \cdot \lambda): L(v \cdot \lambda)]=p_{v w}(1) .
$$

Remark 2.7. If one indexes blocks by $\rho$-antidominant rather than $\rho$-dominant weights, $(2.1)$ takes the form $[M(w \cdot \lambda): L(v \cdot \lambda)]=p_{w_{0} v, w_{0} w}(1)$. Depending on whether one uses the basis elements $C_{w}$ or $C_{w}^{\prime}$ for the Kazhdan-Lusztig basis ${ }^{4}, T_{w}$ or $H_{w}=q^{\ell(w) / 2} T_{w}$ for the natural basis, $q$ or $v=q^{-1 / 2}$ for the formal indeterminate, and $p_{v w}$ or $h_{v w}=q^{-2(\ell(w)-\ell(v))} p_{v w}$ for the Kazhdan-Lusztig polynomials, the precise statement exhibiting the $p_{v w}$ 's as base change coefficients may look different (see also [Soe97, p. 2.6]).

2.3 Gradings We summarise how to pass from $\mathcal{O}_{\lambda}$ to a graded category $\mathcal{O}_{\lambda}^{\mathbf{Z}}$.

The category $\mathcal{O}_{\lambda}$ has a projective generator $P_{\lambda}:=\bigoplus_{w \in W / W_{\lambda}} P(w \cdot \lambda)$, for which we define the algebra $A_{\lambda}:=\operatorname{End}_{\mathcal{O}}\left(P_{\lambda}\right)$. By Morita's theorem [Bas68, Thm. II.1.3], there is an equivalence of categories

$$
\mathcal{O}_{\lambda} \stackrel{\simeq}{\rightarrow} \operatorname{Mod}-A_{\lambda}, \quad M \mapsto \operatorname{Hom}_{\mathcal{O}}\left(P_{\lambda}, M\right) .
$$

There exists a natural grading on $A_{\lambda}$, given as follows [Str03a, $\left.\S \S 1.3,2\right]$.

Theorem 2.8 (Struktursatz) [Soe90, thm. 2]. The functor $\mathbf{V}_{\lambda}: \mathcal{O}_{\lambda} \rightarrow \operatorname{Mod}-\operatorname{End}_{\mathcal{O}}\left(P\left(w_{0} \cdot \lambda\right)\right.$ ), called the combinatoric functor, is fully faithful on projectives.

The algebra $\operatorname{End}_{\mathcal{O}}\left(P\left(w_{0} \cdot \lambda\right)\right)$ can be described explicitly: The ordinary (non-dotted) action of the Weyl group $W$ on $\mathfrak{h}$ gives rise to an action of $W$ on the symmetric algebra $\mathbf{C}\left[\mathfrak{h}^{*}\right]$. Let $\left(\mathbf{C}\left[\mathfrak{h}^{*}\right]_{+}^{W}\right)$ denote its ideal generated by $W$-invariant polynomials without constant term and $C:=\mathbf{C}\left[\mathfrak{h}^{*}\right] /\left(\mathbf{C}\left[\mathfrak{h}^{*}\right]_{+}^{W}\right)$ be the coinvariant algebra of $\mathfrak{g}$.

Theorem 2.9 (Endomorphismensatz) [Soe90, thm. 3]. $\operatorname{End}_{\mathcal{O}}\left(P\left(w_{0} \cdot \lambda\right)\right)$ is isomorphic to the invariant subalgebra $C^{W_{\lambda}}$.

${ }^{4}$ See [KL79, after thm. 1.1] for their relation. 
The images $\mathbf{V P}(w \cdot \lambda)$ of the indecomposable projectives are particular direct summands of the $C$-module $C \otimes_{C^{s_{i_{n}}}} \cdots \otimes_{C^{s_{i_{1}}}} \mathbf{C}$ for a reduced expression $w=s_{i_{1}} \cdots s_{i_{n}}$ [Soe90, thm. 10].

By putting $\mathfrak{h}^{*}$ in degree two, $C\left[\mathfrak{h}^{*}\right]$ becomes an evenly graded algebra. Since $\left(\mathbf{C}\left[\mathfrak{h}^{*}\right]_{+}^{W}\right)$ is a homogeneous ideal, also $\operatorname{End}_{\mathcal{O}}\left(P\left(w_{0} \cdot \lambda\right)\right) \cong C$ is a graded algebra. Tensor products and direct summands of graded modules with indecomposable complement inherit a grading, hence the images $\mathbf{V} P(w \cdot \lambda)$ and the spaces $\operatorname{Hom}_{\mathcal{O}}(P(v \cdot \lambda), P(w \cdot \lambda)) \cong \operatorname{Hom}_{C}(\mathbf{V} P(v \cdot \lambda), \mathbf{V} P(w \cdot \lambda))$ are graded as well, which establishes a grading on $A_{\lambda} \cong \operatorname{End}_{C}\left(\mathbf{V} P_{\lambda}\right)$ [Str03a, thm 2.1]. The equivalence in (2.2) motivates the following:

Definition 2.10 . Let $\mathcal{O}_{\lambda}^{\mathrm{Z}}:=\operatorname{gMod}-A_{\lambda}$ be the category of graded $A_{\lambda}$-modules.

Notation 2.11. We denote the $k$-th graded component of a graded $A$-module $M$ by $M_{k}$. We employ the convention that the grading shift $\langle-\rangle$ shifts upwards; i. e., $M\langle i\rangle_{k}:=M_{k-i}$.

Remark 2.12. A module $M \in \mathcal{O}_{\lambda}$ is gradable if there is a graded module $\tilde{M} \in \mathcal{O}_{\lambda}^{\mathrm{Z}}$ such that forgetting the grading yields $\mathrm{f} \tilde{M}=M$. In particular, simple, Verma and indecomposable projective modules are gradable [Str03a, $\S 2 f$.]. Up to grading shifts and isomorphisms, an indecomposable module has a unique graded lift. In the following, we shall not distinguish notationally between these modules and their graded lifts.

We may fix the shift for the graded lifts of $M(w), L(w)$ and $P(w)$ such that each of these lifts has its non-zero graded component of least degree in degree zero. For a graded module $M$ the multiplicity $\left[M_{i}: L(w)\right]$ of $L(w)$ in $M_{i}$, seen as ungraded modules, therefore is precisely the multiplicity $[M: L(w)\langle i\rangle]$ of graded modules.

Since $A_{\lambda}$ is non-negatively graded, a graded module $M$ is filtered by the submodules $M_{\geq k}:=\bigoplus_{l \geq k} M_{l}$, and its graded components $M_{k}=M_{\geq k} / M_{\geq k+1}$ are subquotients. For Verma and projective modules, these are precisely the Loewy layers [Str03a, p. 302; Irv88, $\S 1.1$. The graded component a simple composition factor belongs to is encoded in the exponents of Kazhdan-Lusztig polynomials:

Theorem 2.13 (generalised KL-theorem) [Irv88, thm. 2; Irv90, cor. 7.1.3; BGS96, thm. 3.1.4 ${ }^{5}$ ]. The composition factor multiplicities in the graded components of Verma modules satisfy

$$
p_{v w}=\sum_{\ell}\left[M(v)_{\ell}: L(w)\right] q^{(\ell(w)-\ell(v)-\ell) / 2}
$$

in other words, each summand $q^{k}$ of $p_{v w}$ corresponds to a factor $L(w)$ in the $\ell=(\ell(w)-$ $\ell(v)-2 k)$-th layer of the Loewy filtration for $M(v)$, with the zeroth layer at the top.

Remark 2.14. If one prefers to work with Soergel's $h_{v w}$ (see remark 2.7), one gets $h_{v w}=$ $\sum_{\ell}\left[M(v)_{\ell}: L(w)\right] v^{\ell}$. If one indexes blocks of $\mathcal{O}$ by antidominant weights instead, $v$ and $w$ have to be replaced by $w_{0} v$ and $w_{0} w$, respectively, and (2.3) becomes $p_{w_{0} v, w_{0} w}=\sum_{\ell}\left[M\left(w_{0} v\right)_{\ell}\right.$ : $\left.L\left(w_{0} w\right)\right] q^{\left(\ell\left(w_{0} v\right)-\ell\left(w_{0} w\right)-\ell\right) / 2}$. This is equivalent to $(2.3)$ since $w_{0}^{2}=e$ and $\ell\left(w_{0} w\right)=\ell\left(w_{0}\right)-$ $\ell(w)$ for all $w$.

A graded analogue of the BGG reciprocity theorem 2.6 holds [Str03a, thm. 7.6].

Definition 2.15. On $\mathcal{O}_{\lambda}^{\mathrm{Z}}$, the graded translation through the $s$-wall $\Theta_{s}$ is uniquely defined by short exact sequences

$$
0 \rightarrow M(w)\langle 1\rangle \stackrel{\eta}{\rightarrow} \Theta_{s} M(w) \rightarrow M(w s) \rightarrow 0 \quad \text { and } \quad \Theta_{s} M(w s)=\Theta_{s} M(w)\langle-1\rangle
$$

${ }^{5}$ Blocks by are indexed by dominant weights, $n_{x y}$ is used for Soergel's $h_{x y}$. 
for $w<w s$. Both adjunction maps are of degree one, and $\Theta_{s}^{2} \cong \Theta_{s}\langle-1\rangle \oplus \Theta_{s}\langle 1\rangle$. Analogously to the non-graded case (see definition 1.5), the graded (co)shuffling functors $\mathrm{Sh}_{s}$ and $\mathrm{Csh}_{s}$ are defined by $\operatorname{Sh}_{s} M=\operatorname{coker}\left(\eta: \operatorname{id}\langle 1\rangle \Rightarrow \Theta_{s}\right)$ and $\operatorname{Csh}_{s} M=\operatorname{coker}\left(\Theta_{s} \Rightarrow \mathrm{id}\langle-1\rangle\right)$.

Remark 2.16. The grading shift $\langle 1\rangle$ renders the Grothendieck group $K_{0}\left(\mathcal{O}_{0}^{\mathbf{Z}}\right)$ becomes a $\mathbf{Z}\left[v^{ \pm 1}\right]$-module by $v[M]:=[M\langle 1\rangle]$. With $v=q^{-1 / 2}$, there is an isomorphism of $\mathbf{Z}\left[v^{ \pm 1}\right]$ modules

$$
K_{0}\left(\mathcal{O}_{0}^{\mathbf{Z}}\left(\mathfrak{s l}_{n}\right)\right) \rightarrow \mathrm{H}_{v}\left(S_{n}\right), \quad[M(w)\langle v\rangle] \mapsto v H_{w}, \quad[P(w)\langle v\rangle] \mapsto v C_{w},
$$

for the Iwahori-Hecke algebra $\mathrm{H}_{v}\left(S_{n}\right)$ (see section 2.2). Explicitly, the basis $\left\{H_{w}\right\}_{w \in W}$ is subject to the relations $H_{w} H_{s}=H_{w s}$ and $H_{w s} H_{s}=H_{w}-\left(v-v^{-1}\right) H_{w s}$ for $w<w s$.

From the defining short exact sequence of $\Theta_{s}$ in (2.4) we see for $w<w s$ that

$$
\begin{aligned}
{\left[\Theta_{s} M(w)\right] } & =v[M(w)]+[M(w s)] \\
{\left[\mathrm{Sh}_{s} M(w)\right] } & =\left[\Theta_{s} M(w)\right]-[M(w)\langle v\rangle]=M(w s) \\
{\left[\mathrm{Sh}_{s} M(w s)\right] } & =\left[\Theta_{s} M(w)\langle-1\rangle\right]-[M(w s)\langle 1\rangle]=[M(w s)]-\left(v-v^{-1}\right)[M(w)],
\end{aligned}
$$

hence $\left[\Theta_{s}\right]$ and $\left[\mathrm{Sh}_{s}\right]$ respectively correspond to the right multiplication $\cdot\left(v+H_{s}\right)$ and $\cdot H_{s}$ under the above isomorphism [Str03a, thm. 7.1]

2.4 Quivers Recall that we defined the algebra $A_{\lambda}:=\operatorname{End}_{\mathcal{O}}\left(P_{\lambda}\right)=\operatorname{End}_{\mathcal{O}}\left(\bigoplus_{w} P(w \cdot \lambda)\right)$ in section 2.3. This algebra $A_{\lambda}$ arises as the path algebra of a quiver as follows.

Definition 2.17. The Ext-quiver $Q_{A}$ associated to a basic algebra $A$ over an alg. closed field has vertices corresponding to the isoclasses of simple $A_{\lambda}$-modules $L$ and $\operatorname{dim} \operatorname{Ext}^{1}\left(L, L^{\prime}\right)$ many arrows $L^{\prime} \leftarrow L$.

Remark 2.18. Equivalently, for a complete set $\left\{e_{i} \mid i \in I\right\}$ of primitive orthogonal idempotents of $A, Q_{A}$ can be defined as the quiver with vertex set $I$ corresponding to the idempotents and $\operatorname{dim}\left(e_{i}(\operatorname{rad} A) e_{j} / e_{i}\left(\operatorname{rad}^{2} A\right) e_{j}\right)$ many arrows $i \leftarrow j$ [ARS95, prop. 1.14; Ben95, prop. 2.4.3; ASS06, lem. 2.12].

By Gabriel's theorem [Ben95, thm. 4.1.7; ASS06, thm. 3.7; Gab80, §4.3], there exists an admissible ideal $\mathfrak{a}$ such that $k Q_{A} / \mathfrak{a} \cong A$. Under this isomorphism, the idempotent $e_{i}$ of $A$ corresponds to the trivial path $\varepsilon_{i}$ in $k Q_{A}$. More generally, every finite dimensional algebra over an alg. closed field is Morita-equivalent to a basic algebra [ASS06, cor. 6.10] and thus to a path algebra quotient.

For the basic $\mathbf{C}$-algebra $A_{\lambda}$ with complete set $\left\{\operatorname{id}_{P(w)}\right\}$ of primitive orthogonal idempotents, we denote the Ext-quiver quiver by $Q_{\lambda}:=Q_{A_{\lambda}}$ and the admissible ideal by $\mathfrak{a}_{\lambda}$, such that $A_{\lambda} \cong Q_{\lambda} / \mathfrak{a}_{\lambda}$.

Notation 2.19. We denote the composition of arrows $v_{2} \stackrel{a}{\leftarrow} v_{1}$ and $v_{3} \stackrel{b}{\leftarrow} v_{2}$ of a quiver by $v_{3} \stackrel{b a}{\longleftarrow} v_{1}$. We denote trivial the path associated to a vertex $v$ by $\varepsilon_{v}$.

The path algebra of any quiver $Q_{\lambda}$ is non-negatively graded by the length of a path in terms of arrows. One can show that $\mathfrak{a}_{\lambda}$ is a homogeneous ideal; hence $A_{\lambda}$ is graded as well. This grading coincides with the grading induced by $\mathbf{V}_{\lambda}$ [Str03a] that we explained in section 2.3. 
To summarise, we have that $\mathcal{O}_{\lambda} \simeq \operatorname{Mod}-A_{\lambda}$ and $\mathcal{O}_{\lambda}^{\mathrm{Z}} \simeq \operatorname{gMod}-A_{\lambda}$. The equivalence $\mathcal{O}_{\lambda} \simeq$ Mod- $A_{\lambda}$ maps indecomposable projectives $P(w)$ in $\mathcal{O}_{\lambda}$ to the indecomposable projectives in $\operatorname{Mod}-A_{\lambda}$; these are precisely the right ideals $\varepsilon_{w} A_{\lambda}$ of all paths ending in $w$ [Bar15, cor. 4.18, rmk. 4.20].

One can choose a $\mathbf{C}$-basis of the projective module $\varepsilon_{w} A_{\lambda}$ consisting of paths ending in $w$, each of which corresponds to a simple composition factor $L(v)\langle i\rangle$ for its source vertex $v$ and length $i$. Hence as a vector space, $\varepsilon_{w} A$ is a direct sum $\varepsilon_{w} A=\bigoplus_{v \in W} \varepsilon_{w} A \varepsilon_{v}$ of graded vector spaces, whose $i$-th graded component has dimension $\operatorname{dim}\left(\varepsilon_{w} A \varepsilon_{v}\right)_{i}=\left[P(w)_{i}: L(v)\langle i\rangle\right]$.

From now on, we shall stick to the principal block $\mathcal{O}_{0}$ and omit all the subscript- $\lambda$ 's from $A$ and $Q$.

2.5 Parabolic subalgebras Let $(W, S)$ be a Coxeter system and $S_{\mathfrak{p}} \subseteq S$ be any subset of the simple reflections of $W$.

Definition 2.20. The associated parabolic subgroup $W_{\mathfrak{p}} \leq W$ is the subgroup $W_{\mathfrak{p}}=\left\langle S_{\mathfrak{p}}\right\rangle$ of $W$. Every left coset in $W_{\mathfrak{p}} \backslash W$ has a unique representative of minimal length [Hum90, §1.10]. We denote the set of such representatives by $W^{\mathfrak{p}}$.

To the quiver $Q$ we associate the full subquiver $Q^{\mathfrak{p}}$ of $Q$ with vertex set $Q^{\mathfrak{p}}=W^{\mathfrak{p}}$ and define the respective algebra $A^{\mathfrak{p}}:=A /\left(\varepsilon_{v}\right)_{v \in W_{\mathfrak{p}}}$. The category $\mathcal{O}_{0}^{\mathfrak{p}}:=\operatorname{Mod}-A^{\mathfrak{p}}$ is equivalent to the smallest Serre subcategory of $\mathcal{O}_{0}^{\mathfrak{p}}$ containing all simple modules $L(w)$ for $w \in W^{\mathfrak{p}}$ $[\mathrm{KM} 16, \S 2]$. The quotient map $A \nrightarrow A^{\mathfrak{p}}$ induces an induction-restriction-adjunction

$$
\operatorname{Ind}^{\mathfrak{p}}: \mathcal{O}_{0} \stackrel{\dashv}{\rightleftarrows} \mathcal{O}_{0}^{\mathfrak{p}}: \operatorname{Res}_{\mathfrak{p}}, \quad M \mapsto M \otimes_{A} A^{\mathfrak{p}}, \quad N_{A} \hookleftarrow N,
$$

where $N_{A}$ denotes the module $N$ on which $A$ acts via $A \rightarrow A^{\mathfrak{p}}$. The functor $\operatorname{Res}_{\mathfrak{p}}$ is fully faithful and thus renders $\mathcal{O}_{0}^{\mathfrak{p}}$ a subcategory of $\mathcal{O}_{0}$. It has a left adjoint $Z^{\mathfrak{p}}:=\operatorname{Ind}^{\mathfrak{p}}$, called Zuckerman functor, and a right adjoint $Z_{\mathfrak{p}}$, called dual Zuckerman functor, which respectively assign to a module $M$ its largest quotient and its largest submodule that have simple composition factors $L(w)$ for $w \in W^{\mathfrak{p}}$ [Maz12, Thm. 6.1]. Let $P^{\mathfrak{p}}(w):=Z^{\mathfrak{p}}(P(w))=$ $\varepsilon_{w} A^{\mathfrak{p}}$ and $M^{\mathfrak{p}}(v):=Z^{\mathfrak{p}}(M(v))$. As notation suggests, the $P^{\mathfrak{p}}(w)$ 's are the indecomposable projectives in $\mathcal{O}_{0}^{\mathfrak{p}}[\operatorname{Maz} 12, \S 4.6]$.

Remark 2.21. The constructions and statements from sections 2.1 to 2.4 have parabolic analogues; namely:

(i) The category $\mathcal{O}_{0}^{\mathfrak{p}}$ has a projective generator $P^{\mathfrak{p}}=\bigoplus_{w \in W^{\mathfrak{p}}} P^{\mathfrak{p}}(w)$.

(ii) The analogously constructed graded version $\mathcal{O}_{0}^{\mathbf{Z}, \mathfrak{p}}$ of $\mathcal{O}_{0}^{\mathfrak{p}}$ contains graded lifts of simples, parabolic Vermas and indecomposable projectives.

(iii) The $P^{\mathfrak{p}}(w)$ 's have parabolic standard filtrations with subquotients isomorphic to $M^{\mathfrak{p}}(v)^{\prime}$ 's.

(iv) The respective multiplicities satisfy a parabolic BGG reciprocity theorem [Roc80, Prop. 4.5, Thm. 6.1].

(v) Parabolic Kazhdan-Lusztig polynomials $p_{v w}^{\mathfrak{p}} \in \mathbf{Z}\left[q^{ \pm 1}\right]$ occur as base change coefficients for parabolic Hecke modules [Deo87, §3; Soe97, rmk. 3.2].

(vi) The generalised Kazhdan-Lusztig theorem 2.13 has the following parabolic analogue for graded lifts of simple, parabolic Verma and indecomposable projective modules [Irv90, Cor. 7.1.3; CC87, Thm. 1.3]:

$$
p_{v w}^{\mathfrak{p}}=\sum_{k \geq 0}\left(P^{\mathfrak{p}}(w)_{\ell}: M^{\mathfrak{p}}(v)\right) q^{(\ell(w)-\ell(v)-\ell) / 2}=\sum_{k \geq 0}\left[M^{\mathfrak{p}}(v)_{\ell}: L(w)\right] q^{(\ell(w)-\ell(v)-\ell) / 2} .
$$


In other words, each summand $q^{k}$ corresponds, for $\ell-(\ell(w)-\ell(v)-2 k)$, to an $M^{\mathfrak{p}}(v)\langle\ell\rangle$ in $P^{\mathfrak{p}}(w)$ and an $L(w)\langle\ell\rangle$ in $M^{\mathfrak{p}}(v)$, with the top of the modules in degree zero.

Remark 2.22. The functor $Z^{\mathfrak{p}}$ commutes with projective functors, in particular with $\Theta_{s}$ [Maz12, Thm. 6.1]. This implies that both $\Theta_{s}$ and $\mathrm{Sh}_{s}$ restrict to $\mathcal{O}_{0}^{\mathfrak{p}}$ and $\mathcal{O}_{0}^{\mathbf{Z}, \mathfrak{p}}$, and the restriction of $\Theta_{s}$ is uniquely characterised by the short exact restrictions

$$
0 \rightarrow M^{\mathfrak{p}}(w)\langle 1\rangle \stackrel{\eta}{\rightarrow} \Theta_{s} \rightarrow M^{\mathfrak{p}}(w s) \rightarrow 0 \quad \text { and } \quad \Theta_{s} M^{\mathfrak{p}}(w s)=\Theta_{s} M^{\mathfrak{p}}(w)\langle-1\rangle
$$

for $w<w s$, of the short exact sequences (1.1) defining $\Theta_{s}$.

Caveat 2.23. The inclusion $\mathcal{O}_{0}^{\mathfrak{p}} \subseteq \mathcal{O}_{0}$ does not preserve projectives, as we shall see in remark 4.9 .

$3 \boldsymbol{B}_{\boldsymbol{n}}$-actions for $\mathfrak{s l}_{\mathbf{2}}$ Consider the Lie algebra $\mathfrak{g}=\mathfrak{s l}_{2}$ and its Weyl group $S_{2}=\{e, s\}$. Since the Kazhdan-Lusztig polynomials for $S_{2}$ are $p_{v w}=\left\{\begin{array}{l}1 \text { if } v \leq w, \\ 0 \text { otherwise, }\end{array}\right.$ the Verma modules and the indecomposable projectives have composition series and standard filtrations

$$
P(e)=M(e)=\left(\begin{array}{c}
L(e) \\
L(s)
\end{array}\right), \quad P(s)=\left(\begin{array}{c}
M(s) \\
M(e)
\end{array}\right)=\left(\begin{array}{c}
L(s) \\
L(e) \\
L(s)
\end{array}\right), \quad M(s)=L(s) .
$$

The category $\mathcal{O}_{0}$ thus is equivalent to $\operatorname{Mod}-A$ for the path algebra quotient $A=\mathbf{C} Q /(b a)$ of the quiver $Q=(a: e \rightleftarrows s: b)$. The arrows $a, b$ of $Q$ and their relations correspond to the unique (up to scalars) non-trivial morphisms $a: P(e) \hookrightarrow P(s)$ and $b: P(s) \rightarrow P(e)$ [Str03a].

3.1 The action of $\mathbf{S h}_{s}$ We obtain the images of the indecomposable projectives and Verma modules under $\mathrm{Sh}_{s}$ as follows:

1. $M(e)=P(e)$ : from the short exact sequence (1.1) we obtain that $\Theta_{s} M(e)=\left(\begin{array}{l}M(s) \\ M(e)\end{array}\right)=$ $P(s)$ and $\mathrm{Sh}_{s} M(e)=M(s)$.

2. $M(s)$ : Up to scalars, there is a unique morphism $M(s) \hookrightarrow M(e)$; hence we obtain that $\mathrm{Sh}_{s} M(s) \cong\left(\begin{array}{c}M(s) \\ M(e) / M(s)\end{array}\right)=\left(\begin{array}{c}L(s) \\ L(e)\end{array}\right)=M^{\vee}(s)$, i. e., the dual Verma module; see [Hum08, §3.2] for the definition.

3. $P(s)$ : using $\Theta_{s}^{2} \cong \Theta_{s} \oplus \Theta_{s}$, it follows from $P(s)=\Theta_{s} M(e)$ that $\operatorname{Sh}_{s} P(s) \cong P(s)$.

From the naturality diagram

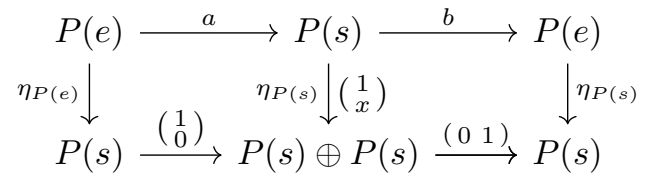

of $\eta$ : id $\Rightarrow \Theta_{s}$ we get that the morphisms $a: P(e) \hookrightarrow P(s)$ and $b: P(s) \rightarrow P(e)$ have images

$$
\mathrm{Sh}_{s} a: M(s) \hookrightarrow P(s) \text { and } \mathrm{Sh}_{s} b: P(s) \rightarrow M(s) .
$$

Similar arguments show that $\operatorname{Csh}_{s} P(s) \cong P(s)$ and $\mathbf{R} \operatorname{Csh}_{s} P(e)$ is the mapping cone $\mathbf{R} \mathrm{Csh}_{s} P(e) \simeq\{P(s) \rightarrow P(e)\}$ (recall the notation of mapping cones from Notation 1.16); so we have

$$
\begin{aligned}
& \mathbf{L} \operatorname{Sh}_{s} P(e)=\left\{P(e) \rightarrow \underset{0}{P(s)} \simeq \simeq M(s) \quad \mathbf{R} \operatorname{Csh}_{s} P(e)=\left\{\begin{array}{c}
P(s) \rightarrow P(e) \\
0
\end{array}\right.\right. \\
& \mathbf{L} \mathrm{Sh}_{s} P(s)=P(s) \quad \mathbf{R} \mathrm{Csh}_{s} P(s)=P(s),
\end{aligned}
$$


and in terms of the projective resolution $\{P(e) \rightarrow P(s)\}$ of $M(s)$, the morphisms from (3.3) become

$$
\begin{aligned}
& \mathbf{L S h}_{s} a:\left\{\begin{array}{r}
\{P(e) \rightarrow P(s)\} \\
\downarrow a b \\
P(s),
\end{array} \quad \mathbf{L S h}_{s} b:\left\{\begin{array}{r}
P(s) \\
\downarrow \text { id } \\
\{P(e) \rightarrow P(s)\},
\end{array}\right.\right. \\
& \mathbf{R} \operatorname{Csh}_{s} a:\left\{\begin{array}{l}
\{P(s) \rightarrow P(s)\} \\
\downarrow \text { id } \\
P(s),
\end{array} \quad \mathbf{R C s h}_{s} b:\left\{\begin{array}{c}
P(s) \\
\downarrow a b \\
\{P(s) \rightarrow P(s)\} .
\end{array}\right.\right.
\end{aligned}
$$

Since $\mathcal{O}_{0}$ has finite global dimension [Hum08, prop. 6.9], $D^{\mathrm{b}}\left(\mathcal{O}_{0}\right)$ is equivalent to the bounded homotopy category of projectives (cf. [Wei94, thm. 10.4.8]) and therefore generated as a triangulated category by the indecomposable projectives; i. e., the smallest triangulated subcategory of $D^{\mathrm{b}}\left(\mathcal{O}_{0}\right)$ containing the indecomposable projectives is $D^{\mathrm{b}}\left(\mathcal{O}_{0}\right)$ itself $[\mathrm{Kra} 07$, §3.3]. These above data thus suffices to determine $\mathbf{L} \mathrm{Sh}_{s}$. Phrasing the observations differently, we have shown the following:

Proposition 3.1. The action on $D^{\mathrm{b}}\left(\mathcal{O}_{0, \mathfrak{s}_{2}}\right)$ of the Artin braid group $B_{1}$ (which is an infinite cyclic group) by $s \mapsto \mathbf{L} \mathrm{Sh}_{s}$ and $s^{-1} \mapsto \mathbf{R} \mathbf{C s h}_{s}$ is given explicitly by

$$
\begin{aligned}
& B_{1} \longrightarrow \operatorname{Aut}\left(D^{\mathrm{b}}\left(\mathcal{O}_{0}\right)\right) \\
& s^{n} \longmapsto P(s) \mapsto P(s) \text {, }
\end{aligned}
$$

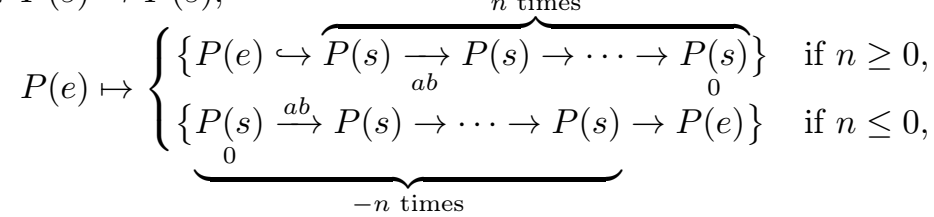

with homological degree 0 as indicated.

Example 3.2. To illustrate why this proposition is clear from what we derived before, let us compute the action of, say, $s^{2}$, which acts by $\mathbf{L} \operatorname{Sh}_{s}^{2}$. In $D^{\mathrm{b}}\left(\mathcal{O}_{0}\right)$, the module $M(s)$ is isomorphic to its projective resolution $\{P(e) \stackrel{a}{\rightarrow} P(s)\}$, to which we apply $\mathbf{L} \mathrm{Sh}_{s}^{2}$. From the images in (3.3) and (3.4) we get that $\mathbf{L} \mathrm{Sh}_{s} M(s)$ is isomorphic to the double complex

$$
\mathbf{L S h}_{s} M(s) \simeq\left\{\begin{array}{l}
P(e) \\
\downarrow b \\
P(s) \stackrel{\text { id }}{\longrightarrow} P(s)
\end{array}\right\} \simeq\{P(e) \rightarrow P(s) \rightarrow P(s)\} .
$$

Applying $\mathbf{L} \mathrm{Sh}_{s}$ once again shows that $\mathbf{L} \mathrm{Sh}_{s} M(s) \simeq\{P(e) \rightarrow P(s) \rightarrow P(s) \rightarrow \underset{0}{P(s)}$.

3.2 Spherical objects To check that a spherelike object is spherical indeed, i. e., that the composition pairing from the Calabi-Yau-property (definition 1.12(S3)) is non-degenerate, it suffices to check non-degeneracy w.r.t. indecomposable projectives:

Lemma 3.3. Let $\mathcal{C}$ be an abelian category of finite global dimension and $E \in D^{\mathrm{b}}(\mathcal{C})$ a $d$-spherelike object. If the composition pairing $\operatorname{Hom}_{D^{\mathrm{b}}}^{i}(P, E) \otimes \operatorname{Hom}_{D^{\mathrm{b}}}^{d-i}(P, E) \rightarrow k x$ is nondegenerate for all $i$ and all indecomposable projective objects $P \in \mathcal{C}$, seen as complexes in $D^{\mathrm{b}}(\mathcal{C})$ concentrated in a single degree, then $\operatorname{Hom}_{D^{\mathrm{b}}}^{i}(F, E) \otimes \operatorname{Hom}_{D^{\mathrm{b}}}^{d-i}(F, E) \rightarrow k x$ is non-degeneate for all $i$ and $F \in D^{\mathrm{b}}(\mathcal{C})$. 
Proof. Since $\mathcal{C}$ is of finite global dimension, every object of $D^{\mathrm{b}}(\mathcal{C})$ is quasi-isomorphic to a bounded complex of projectives; in other words, for the full subcateogires $S_{0}:=\mathcal{C}$-Proj and $S_{s}:=\left\{\right.$ cone $\left.f \mid f \in S_{s-1}\right\}$ of $D^{\mathrm{b}}(\mathcal{C})$, we have $D^{\mathrm{b}}(\mathcal{C})=\bigcup_{s>0} S_{s}$. Assume for some $s \geq 0$ that the pairing $\operatorname{Hom}_{D^{\mathrm{b}}}^{i}(F, E) \otimes \operatorname{Hom}_{D^{\mathrm{b}}}^{d-i}(F, E) \rightarrow k x$ is non-degenerate whenever $F \in S_{s}$. For a distinguished triangle $F^{\prime} \stackrel{f}{\rightarrow} F \rightarrow F^{\prime \prime} \rightarrow F^{\prime}[-1]$ with $F, F^{\prime} \in S_{s}$, we thus get that in the diagram

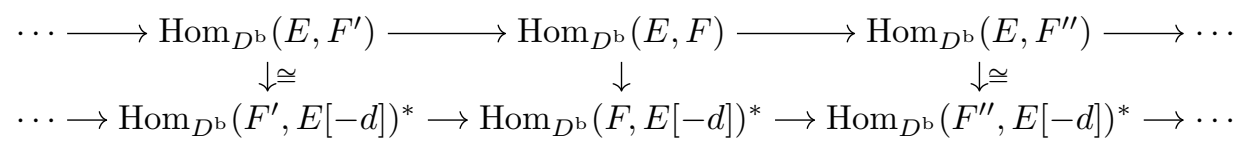

whose rows are long exact sequences, the indicated vertical maps are isomorphisms. By the five lemma, it follows that also $\operatorname{Hom}_{D^{\mathrm{b}}}^{i}\left(F^{\prime \prime}, E\right) \otimes \operatorname{Hom}_{D^{\mathrm{b}}}^{d-i}\left(F^{\prime \prime}, E\right) \rightarrow k x$ is non-degenerate. The claim follows by induction.

Lemma 3.4. $P(s)$ is a 0 -spherical and $L(e)$ is a 2-spherical object of $D^{\mathrm{b}}\left(\mathcal{O}_{0}\right)$ for $\mathfrak{s l}_{2}$.

Proof. To see that $P(s)$ is 0 -spherical, note that the non-trivial endomorphism $x:=a b$ of $P(s)$ satisfies $x^{2}=0$ and $\operatorname{End}_{\mathcal{O}}(P(s)) \cong \mathbf{C}[x] /(x)^{2}$, so $P(s)$ is 0 -spherelike. Since $P(e)$ and $P(s)$ generate $D^{\mathrm{b}}\left(\mathcal{O}_{0}\right)$ and since $x$ factors through $P(e)$, the composition pairing is non-degenerate, hence $P(s)$ is spherical.

To see that $L(e)$ is 2 -spherical, consider its projective resolution $\{P(e) \rightarrow P(s) \rightarrow P(e)\}$. Apart from the identity, the only non-trivial graded endomorphisms of this resolution are (scalar multiples of)

$$
\left(\begin{array}{c}
P(e) \rightarrow P(s) \rightarrow P(e) \\
\downarrow \\
P(e) \rightarrow P(s) \rightarrow P(e)
\end{array}\right),\left(\begin{array}{c}
P(e) \rightarrow P(s) \rightarrow P(e) \\
P(e) \rightarrow P(s) \rightarrow P(e)
\end{array}\right),\left(\begin{array}{c}
P(e) \rightarrow P(s) \rightarrow P(e) \\
\begin{array}{l}
P(e) \rightarrow P(s) \rightarrow P(e) \\
=: x
\end{array}
\end{array}\right)
$$

of which the first two are null-homotopic with the indicated chain homotopies. Therefore, $\operatorname{End}_{D^{\mathrm{b}}(\mathcal{O})}(L(e))=\mathbf{C}[x] /\left(x^{2}\right)$ with $x$ of degree two, which renders $L(e) 2$-spherelike. The endomorphism $x$ factors through $P(e)$, and $\operatorname{Hom}_{D^{\mathrm{b}}(\mathcal{O})}^{*}(P(s), L(e))=0=\operatorname{Hom}_{D^{\mathrm{b}}(\mathcal{O})}^{*}(L(e), P(s))$, hence the composition pairing is non-degenerate.

Remark 3.5. Simple modules in $\mathcal{O}_{0}$ only have trivial self-extensions [Hum08, Prop. 3.1] and thus cannot be 0 or 1-spherical.

3.3 Spherically twisting by $\boldsymbol{P}(s)$ The images of projectives under the cotwisting functor $T_{P(s)}^{\prime}$ are

$$
\begin{aligned}
& T_{P(s)}^{\prime} P(s)=\left\{\left(\begin{array}{l}
1 \\
x
\end{array}\right): \underset{0}{P(s)} \rightarrow P(s) \oplus P(s)\right\} \simeq P(s)[1] \stackrel{(3.4)}{=} \mathrm{Sh}_{s} P(s)[1], \\
& T_{P(s)}^{\prime} P(e)=\{a: P(e) \rightarrow P(s)\} \quad \simeq M(s)[1]=\operatorname{Sh}_{s} P(e)[1] .
\end{aligned}
$$

This is a first step towards a proof of the following:

Theorem 3.6. There is a natural isomorphism $\mathbf{L} \mathrm{Sh}_{s}[1] \cong T_{P(s)}^{\prime}$ of autoequivalences of $D^{\mathrm{b}}\left(\mathcal{O}_{0}\left(\mathfrak{s l}_{2}\right)\right)$. 
Lemma 3.7 (Morita). Let $A$ and $B$ be rings. Any right exact functor $F$ : Mod- $A \rightarrow$ Mod- $B$ that preserves arbitrary direct sums is isomorphic to tensoring with the $A$ - $B$-bimodule $F A$ [Bas68, Thm. II.2.3].

Corollary 3.8. For abelian categories $\mathcal{A}$ and $\mathcal{B}$ with projective generators $P_{\mathcal{A}}$ and $P_{\mathcal{B}}$, Morita's theorem (2.2) allows us to identify $\mathcal{A}$ and $\mathcal{B}$ with $\operatorname{Mod}-\operatorname{End}_{\mathcal{A}}\left(P_{\mathcal{A}}\right)$ and $\operatorname{Mod}-\operatorname{End}_{\mathcal{B}}\left(P_{\mathcal{B}}\right)$ respectively. Then any right exact functor $F: \mathcal{A} \rightarrow \mathcal{B}$ that commutes with arbitrary direct sums can be identified with the functor

$$
-\otimes_{\operatorname{End}_{\mathcal{A}}\left(P_{\mathcal{A}}\right)} M_{F}: \operatorname{Mod}-\operatorname{End}_{\mathcal{A}}\left(P_{\mathcal{A}}\right) \rightarrow \operatorname{Mod}-\operatorname{End}_{\mathcal{B}}\left(P_{\mathcal{B}}\right)
$$

where $M_{F}:=\operatorname{Hom}_{\mathcal{B}}\left(P_{\mathcal{B}}, F P_{\mathcal{A}}\right)$ is an $\operatorname{End}_{\mathcal{A}}\left(P_{\mathcal{A}}\right)$-End $\operatorname{End}_{\mathcal{B}}\left(P_{\mathcal{B}}\right)$-bimodule on which elements $a \in \operatorname{End}_{\mathcal{A}}\left(P_{\mathcal{A}}\right)$ and $b \in \operatorname{End}_{\mathcal{B}}\left(P_{\mathcal{B}}\right)$ act by $a . f . b=F a \circ f \circ b$ for $f \in M_{F}$.

Proof of theorem 3.6. By the corollary there are natural isomorpisms

$$
\begin{aligned}
& \mathbf{L} \operatorname{Sh}_{s}[1] \stackrel{\text { def }}{=}\left\{\operatorname{id}_{0} \Rightarrow \Theta_{s}\right\} \cong-\otimes_{A}\left\{A \rightarrow M_{\Theta_{s}}\right\} \\
& \left.T_{P(s)}^{\prime} \stackrel{\text { def }}{=} \underset{0}{\operatorname{id}_{\mathcal{O}} \Rightarrow \Xi_{P(s)}^{\prime}}\right\} \cong-\otimes_{A}\left\{A \rightarrow M_{\Xi_{P(s)}^{\prime}}\right\},
\end{aligned}
$$

such that it suffices to show $M_{\Theta_{s}} \cong M_{\Xi_{P(s)}^{\prime}}$. Recall that $\Xi_{P(s)}^{\prime}=\operatorname{lin}_{\mathbf{C}}\left(\operatorname{hom}_{\mathcal{O}}(-, P(s)), P(s)\right)$. By finite dimensionality, there is an isomorphism $M_{\Xi_{P(s)}^{\prime}} \cong P(s)^{*} \otimes_{\mathbf{C}} P(s)$ of $A$ - $A$-bimodules. Consider the canonical vector space basis $\left\{\varepsilon_{s}, s \leftarrow e, s \leftarrow e \leftarrow s\right\}$ of $P(s)=\varepsilon_{s} A$ and the dual basis of $P(s)^{*}$. A basis of $M_{\Xi_{P(s)}^{\prime}}$ is given by the nine pairwise tensor products in the schematic

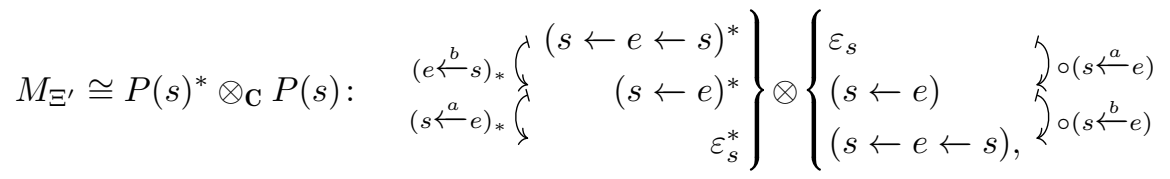

with the indicated action on basis vectors. To describe the $A$ - $A$-bimodule action on $M_{\Theta_{s}}=$ $\operatorname{Hom}_{A}\left(P, \Theta_{s} P\right)$ in terms of a vector space basis, we introduce the following notation. Recall that $P=P(e) \oplus P(s)$ and $\Theta_{s} P \cong P(s)^{\oplus 3}$. We enumerate the summands of $\Theta_{s} P=$ $P(s)_{1} \oplus P(s)_{2} \oplus P(s)_{3}$. We then abbreviate, e. g., the morphism $\left(\begin{array}{ll}0 & 0 \\ 0 & 0 \\ 0 & x\end{array}\right) \in \operatorname{Hom}_{A}\left(P, \Theta_{s} P\right)$, by $P(s)_{3} \stackrel{x}{\leftarrow} P(s)$. For $\Theta_{s}$, the naturality diagram (3.2) of $\eta$ : id $\Rightarrow \Theta_{s}$ shows that the images under $\Theta_{s}$ of the morphisms $a$ and $b$ generating $\operatorname{End}_{\mathcal{C}}(P)$ are

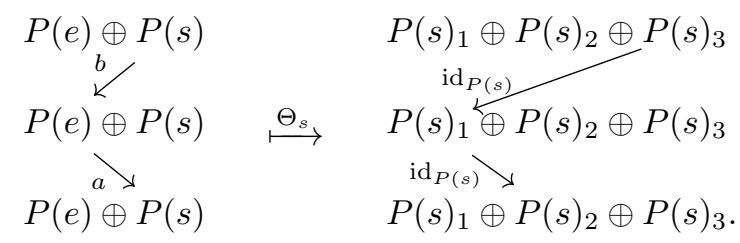

A vector space basis of $M_{\Theta_{s}}$ is given by the nine ways to map a summand $P(w)$ of $P$ from the right of the following schematic to a summand $P(s)_{i}$ of $\Theta_{s} P(s)$ on the left of

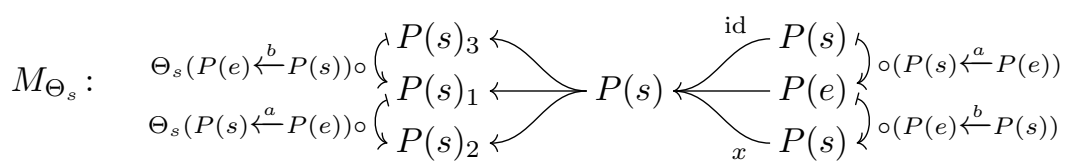


with the bimodule action as indicated. Comparison of (3.8) and (3.10) shows that the obvious isomorphism $M_{\Theta_{s}} \cong M_{\Xi_{P(s)}^{\prime}}$ of vector spaces is an isomorphism of $A$ - $A$-bimodules.

3.4 Spherically twisting by $\boldsymbol{L}(\boldsymbol{e})$ We first note that $\mathbf{L} \mathrm{Sh}_{s} L(e)=L(e)[-1]$; namely, from the images in (3.4) we get that the projective resolution $\{P(e) \rightarrow P(s) \rightarrow P(e)\}$ of $L(e)$ is mapped under $\mathbf{L} \mathrm{Sh}_{s} L(e)$ to $\{M(s) \rightarrow P(s) \rightarrow \underset{0}{M}(s)\}$, which is quasi-isomorphic to $L(e)[-1]$.

Since $L(e)$ is 2-spherical as we have seen in section 3.2, remark 1.17 implies that if there is any isomorphism $T_{L(e)}^{\prime} \cong \mathbf{L} \operatorname{Sh}_{s}[k]$, then the shift $k$ must be zero. We shall now show that

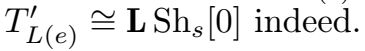

Computing $T_{L(e)}^{\prime}$ involves $\operatorname{hom}_{\mathcal{O}}^{\bullet}(-, L(e))$, for which we employ the following notation. Between angle brackets, we write down $\mathbf{C}$-bases for the homological components of hom $\mathfrak{\mathcal { O }}(-, L(e))$. Every basis element, which is a morphism of complexes, is written with the codomain and the (shifted) domain written horizontally and the map of complexes vertically. The horizontal arrows between the $\langle\cdots\rangle$ 's carry matrix representations of the boundary map of hom $\bullet_{\mathcal{O}}(-, L(e))$ w.r.t. these bases. From the projective resolution $L(e) \simeq\{P(e) \rightarrow P(s) \rightarrow P(e)\}$, we obtain

$$
\begin{aligned}
& \operatorname{hom}_{\mathcal{O}}^{\bullet}(P(s), L(e)) \\
& =\left\{\left\langle\begin{array}{l}
P(s) \\
b \downarrow \\
P(e) \rightarrow P(s) \rightarrow P(e)
\end{array}\right\rangle \stackrel{\left(\begin{array}{l}
0 \\
1
\end{array}\right)}{\longrightarrow}\left\langle\begin{array}{c}
P(s) \\
\operatorname{id}, x \downarrow \\
P(e) \rightarrow P(s) \rightarrow P(e)
\end{array}\right\rangle \stackrel{(1,0)}{\longrightarrow}\left\langle\begin{array}{r}
P(s) \\
\downarrow b \\
P(e) \rightarrow P(s) \rightarrow P(e)
\end{array}\right\rangle\right\} \\
& \simeq 0 \text {, } \\
& \operatorname{hom}_{\mathcal{O}}^{\bullet}(L(e), P(s)) \\
& =\left\{\left\langle\begin{array}{c}
P(e) \rightarrow P(s) \rightarrow P(e) \\
b \downarrow \\
P(s)
\end{array}\right\rangle \stackrel{\left(\begin{array}{l}
0 \\
1
\end{array}\right)}{\longrightarrow}\left\langle\begin{array}{c}
P(e) \rightarrow P(s) \rightarrow P(e) \\
\operatorname{id}, x \downarrow \\
P(s)
\end{array}\right\rangle \stackrel{(1,0)}{\longrightarrow}\left\langle\begin{array}{c}
P(e) \rightarrow P(s) \rightarrow P(e) \\
b \downarrow \\
P(s)
\end{array}\right\rangle\right\} \\
& \simeq 0 \text {, }
\end{aligned}
$$

We thus get immediately from the definition 1.13 of $T^{\prime}$ and $T$ that $\Xi_{L(e)}^{\prime} P(s)=0=\Xi_{L(e)} P(s)$ and thus that $T_{L(e)}^{\prime} P(s)=P(s)=T_{L(e)} P(s)$. For $P(e)$, we obtain

$$
\begin{aligned}
& \operatorname{hom}_{\mathcal{O}}(P(e), L(e))
\end{aligned}
$$

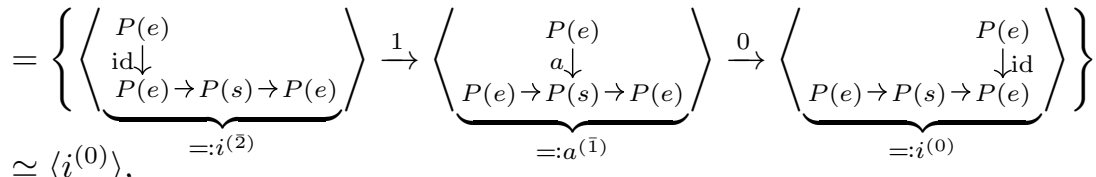

$$
\begin{aligned}
& \operatorname{hom}_{\mathcal{O}}^{\bullet}(L(e), P(e))
\end{aligned}
$$

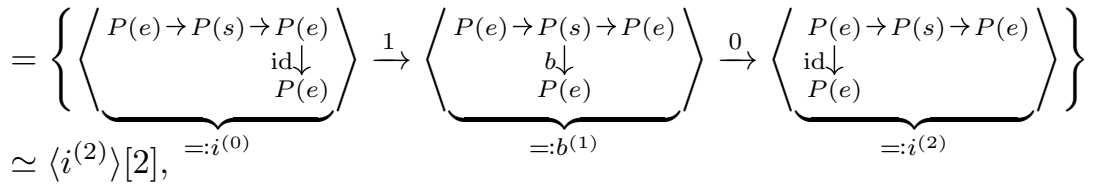

We denote the morphisms of complexes as indicated in the above formulae. Before we compute the images of $P(e)$ under $T_{L(e)}^{\prime}$ and $T_{L(e)}$, we state the following: 
Lemma 3.9 (Elimination of trivial summands). In any abelian category, if the map $d$ in the chain complex in the first line of the diagram

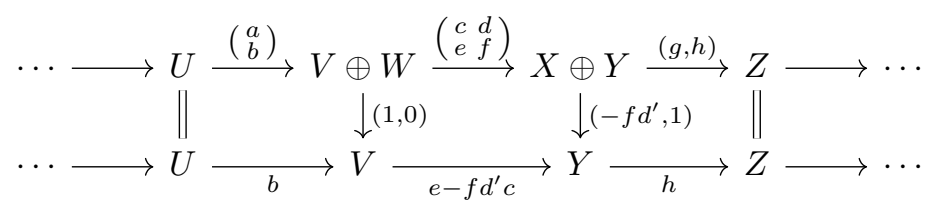

is an isomorphism with inverse $d^{\prime}$, the second line is a chain complex and the vertical map is a quasi-isomorphism.

We now compute the images $T_{L(e)}^{\prime} P(e)$ and $T_{L(e)} P(e)$ parallelly in two columns. These are the respective total complexes of the triple complexes

$$
\begin{aligned}
& T_{L(e)}^{\prime} P(e) \\
&=\{\begin{array}{l}
P(e) \stackrel{e v^{\prime}}{\longrightarrow} \underbrace{\operatorname{lin}_{\mathbf{C}}\left(\operatorname{hom}_{\mathcal{O}}^{\bullet}(P(e), L(e)), L(e)\right)}_{\Xi_{L(e)}^{\prime}}\}
\end{array}=\underbrace{\left\{\operatorname{hom}_{\mathcal{O}}^{\bullet}(L(e), P(e)) \otimes L(e)\right.}_{\Xi_{L(e)} P(e)} \stackrel{e v}{\rightarrow} P(e)\} \\
& 0
\end{aligned}
$$

with the gray $P(e)$ in degree 0 . Recall from remark 1.11 that the double complexes $\operatorname{lin}_{\mathbf{C}}\left(\operatorname{hom}_{\mathcal{O}}^{\bullet}(P(e), L(e)), L(e)\right)$ and $\operatorname{hom}_{\mathcal{O}}^{\bullet}(P(e), L(e), P(e)) \otimes L(e)$, are sums of shifted copies of $L(e)$, indexed by basis elements from (3.13) and (3.14). Working this out gives that $T_{L(e)}^{\prime} P(e)$ and $T_{L(e)} P(e)$ respectively are the triple complexes
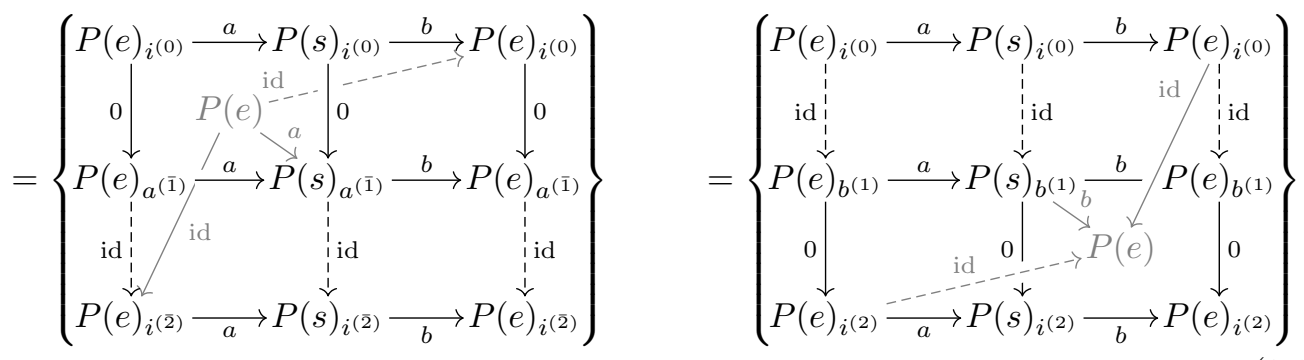

still with the gray $P(e)$ in degree zero. Here, the black $P(-)$ 's are indexed by basis elements from (3.13) and (3.14), according to the copy of $L(e)$ they belong to. Since $\operatorname{hom}_{\mathcal{O}}(P(e), L(e))$, $\operatorname{hom}_{\mathcal{O}}^{\bullet}(L(e), P(e))$ and $L(e)$ are respectively concentrated in degrees -2 to 0,0 to 2 and -2 to 0 , the two double complexes $\Xi_{L(e)}^{\prime} P(e)$ and $\Xi_{L(e)} P(e)$ printed in black have their degree-zero summands on the secondary diagonal. According to remark 1.14, the non-zero components of the (co)evaluation maps therefore consist of the gray morphisms. Using the elimination lemma 3.9, we may eliminate all summands adjacent to one of the dashed identity morphisms; this shows that there are quasi-isomorphisms

$$
\begin{aligned}
& \simeq\{P(e) \stackrel{a}{\rightarrow} \underset{0}{P(s)}\}, \\
& \simeq \underset{0}{\{P(s) \stackrel{b}{\rightarrow} P(e)\}} \\
& \simeq \mathbf{L} \operatorname{Sh}_{s} P(e), \\
& \simeq \mathbf{R} \operatorname{Csh}_{s} P(e),
\end{aligned}
$$

by comparison with (3.4). Hence, we already have shown half of the following: 
Theorem 3.10. There is a natural isomorphism $T_{L(e)}^{\prime} \cong \mathbf{L} \mathrm{Sh}_{s}$.

Proof. To show that both functors are isomorphic we have yet to show that the morphisms $\operatorname{id}_{P(e)}, \operatorname{id}_{P(s)}, a$ and $b$ generating $\operatorname{End}_{\mathcal{O}}(P)$ have isomorphic images under both functors. Recall the images $\mathrm{Sh}_{s} a: M(s) \hookrightarrow P(s)$ and $\mathrm{Sh}_{s} b: P(s) \rightarrow M(s)$ from (3.3). Recall from remark 1.14 how the maps $a: P(e) \rightarrow P(s)$ and $b: P(s) \rightarrow P(e)$ induce morphisms $a_{*}$ : $\Xi_{L(e)}^{\prime} P(e) \rightarrow \Xi_{L(e)}^{\prime} P(s)$ and $b_{*}: \Xi_{L(e)}^{\prime} P(s) \rightarrow \Xi_{L(e)}^{\prime} P(s)$. Since

$$
\begin{aligned}
a^{*}: \operatorname{hom}_{\mathcal{O}}(P(s), L(e)) \rightleftarrows & \operatorname{hom}_{\mathcal{O}}^{\bullet}(P(e), L(e)): b^{*} \\
b^{(0)}, x^{(\overline{1})}, b^{(\overline{2})} \longmapsto 0, & b^{(0)} \longleftrightarrow i^{(0)} \\
i^{(\overline{1})} \longmapsto a^{(\overline{1})} & x^{(\overline{1})} \longleftrightarrow a^{(\overline{1})} \\
& b^{(\overline{2})} \longleftrightarrow i^{(\overline{2})},
\end{aligned}
$$

the morphisms $a_{*}$ and $b_{*}$ act on an element $m_{f}$ of a summand $L(e)[\operatorname{deg} f]_{f}$ of $\Xi_{L(e)}^{\prime} P(e)$ or $\Xi_{L(e)}^{\prime} P(e)$ indexed by a basis element $f$ by

$$
\begin{aligned}
& a_{*}: \Xi_{L(e)}^{\prime} P(e) \rightleftarrows \Xi_{L(e)}^{\prime} P(s): b_{*} \\
& m_{i^{(0)}}, m_{i^{(\overline{2})}} \longmapsto 0 \quad m_{i^{(0)}} \leftrightarrow m_{b^{(0)}} \quad 0 \leftrightarrow m_{i^{(\overline{1})}} \\
& m_{a^{(\overline{1})}} \longmapsto m_{i^{(\overline{1})}} \quad m_{a^{(\overline{1})}} \leftrightarrow m_{x^{(\overline{1})}} \quad m_{i^{(\overline{2})}} \leftrightarrow m_{b^{(\overline{2})}} .
\end{aligned}
$$

The maps $T_{L(e)}^{\prime} a$ and $T_{L(e)}^{\prime} b$ are therefore quasi-isomorphic to

$$
\begin{aligned}
& T_{L(e)}^{\prime} P(s) \simeq\{P(s) \stackrel{e v^{\prime}}{\longrightarrow} \overbrace{L(e)_{b^{(0)}} \oplus L(e)[1]_{i^{(\overline{1})}} \oplus L(e)[1]_{x^{(\overline{1})}} \oplus L(e)[2]_{b^{(\overline{2})}}}^{\Xi_{L(e)}^{\prime} P(s)}\}
\end{aligned}
$$

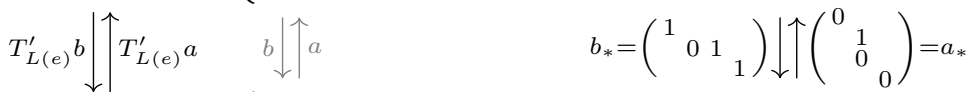

$$
\begin{aligned}
& T_{L(e)}^{\prime} P(e) \simeq\{P(e) \longrightarrow v^{\prime} \longrightarrow \underbrace{L(e)_{i^{(0)}} \oplus L(e)[1]_{a^{(\overline{1})}} \oplus L(e)[2]_{i^{(\overline{2})}}}_{\Xi_{L(e)}^{\prime} P(e)}\} .
\end{aligned}
$$

Consider again the left triple complex in (3.15), whose black printed "front layer" represents $\Xi_{L(e)}^{\prime} P(e)$, and use the elimination lemma 3.9 to eliminate only the bottom three identities $P(-)_{\left.a^{(\overline{1}}\right)} \rightarrow P(-)_{i(\overline{(})}$. Similarly, a triple complex representing $T_{L(e)}^{\prime} P(s)$ can be obtained from (3.11). We obtain that $T_{L(e)}^{\prime} a$ and $T_{L(e)}^{\prime} b$ are represented by the maps

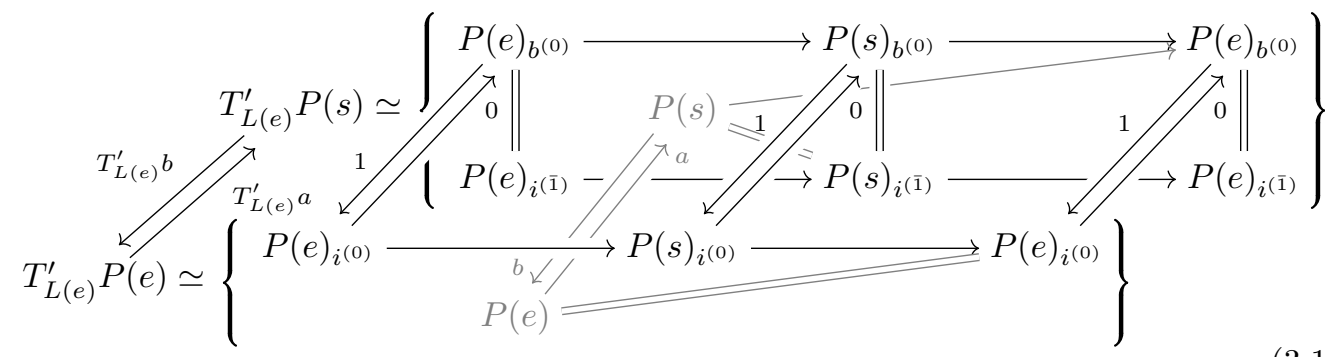


between the triple complexes representing $T_{L(e)}^{\prime} P(s)$ and $T_{L(e)}^{\prime} P(e)$. We pass to the total complexes of (3.16) and use the elimination lemma 3.9 to choose quasi-isomorphic replacements

$$
\begin{aligned}
& P(s)
\end{aligned}
$$

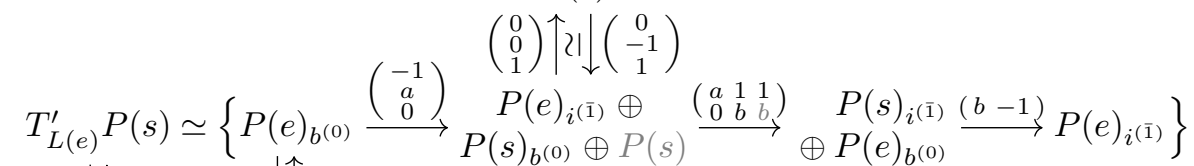

$$
\begin{aligned}
& \left.T_{L(e)}^{\prime} b\left|\uparrow_{T_{L(e)}^{\prime} a} \quad 1 \downarrow\right|\right|_{0} \\
& T_{L(e)}^{\prime} P(e) \simeq\left\{P(e)_{i^{(0)}} \stackrel{\left(\begin{array}{l}
a \\
0
\end{array}\right)}{\longrightarrow} P(s)_{i^{(0)}} \oplus P(e) \underset{(b}{(b)} P(e)_{i^{(0)}}\right\} \\
& \left(\begin{array}{c}
\text { can } \\
-\operatorname{can}
\end{array}\right)\lceil 21 \downarrow(\operatorname{can} 0) \\
& M(s) \text {. }
\end{aligned}
$$

This shows that $T_{L(e)}^{\prime} b=\mathbf{L} \mathrm{Sh}_{s} b: P(s) \rightarrow M(s)$ and $T_{L(e)}^{\prime} a=\mathrm{Sh}_{s} a: M(s) \hookrightarrow P(s)$ indeed. Since all morphisms in $D^{\mathrm{b}}\left(\mathcal{O}_{0}\right)$ are generated by $a$ and $b$, this proves the statement.

$4 \boldsymbol{B}_{n}$-actions for $\mathfrak{s l}_{\mathbf{3}}$ and $\mathfrak{s l}_{\boldsymbol{n}}$ The Lie algebra $\mathfrak{s l}_{3}$ has as its Weyl group the symmetric group $S_{3}=\left\{e, s, t, s t, t s, w_{0}\right\}$. A quiver $Q_{\mathfrak{s t}_{3}}$, which has vertices indexed by $S_{3}$ and unique edges $w \leftrightarrows w s$ for all $w<w s$, and a homogeneous ideal $\mathfrak{a}_{\mathfrak{s l}_{3}}$ of $\mathbf{C} Q_{\mathfrak{s l}_{3}}$ such that $\mathcal{O}_{0, \mathfrak{s l}_{3}} \simeq \operatorname{Mod}-A_{\mathfrak{s} r_{3}}$ for the algebra $A_{\mathfrak{s l}_{3}}=\mathbf{C} Q_{\mathfrak{s l}_{3}} / \mathfrak{a}_{\mathfrak{s l}_{3}}$ is provided in [Str03b; Mar06].

One sees that $Q_{\mathfrak{s l}_{2}}$ is a full subquiver of $Q_{\mathfrak{s l}_{3}}$ and $\mathfrak{a}_{\mathfrak{s l}_{3}} \cap \mathbf{C} Q_{\mathfrak{s l}_{2}}=\mathfrak{a}_{\mathfrak{s l}_{2}}$. The inclusion $A_{\mathfrak{s l}_{2}} \hookrightarrow A_{\mathfrak{S l}_{3}}$ thus induced gives rise to an adjoint pair of functors

$$
\begin{aligned}
\operatorname{Re}_{\mathfrak{s l}}^{\mathfrak{s l}} \mathfrak{l}_{3} & \mathcal{O}_{0, \mathfrak{s l}_{3}} \stackrel{\dashv}{\rightleftarrows} \mathcal{O}_{0, \mathfrak{s l}_{2}}: \operatorname{Ind}_{\mathfrak{s l}_{2}}^{\mathfrak{s l}_{3}}, \\
P(e), P(t) & \mapsto P(e), \quad P(e) \leftrightarrow P(e), \\
P(s), P(s t), P(t s), P\left(w_{0}\right) & \mapsto P(s), \quad P(s) \leftrightarrow P(s),
\end{aligned}
$$

which turns $\mathcal{O}_{0, \mathfrak{s l}_{2}}$ into a full subcategory of $\mathcal{O}_{0, \mathfrak{s l}_{3}}$. In particular, $\operatorname{End}_{\mathcal{O}_{0, \mathfrak{s}_{3}}}(P(s)) \cong \mathbf{C}[x] /\left(x^{2}\right)$ and $P(s)$ is 0 -spherelike also in $\mathcal{O}_{0, \mathfrak{s l}_{3}}$.

Caveat 4.1. The Calabi-Yau property from definition 1.12(S3) is not "local", in the sense that an object can lose this property in a larger ambient category. For instance, there are non-trivial morphisms $P(s) \rightarrow P(t)$ and $P(t) \rightarrow P(s)$ in $\mathcal{O}_{0, \mathfrak{s}_{3}}$ whose composition is zero, so $P(s)$ cannot be spherical. We shall present two possible remedies in this section.

4.1 Spherical subcategories Consider a $k$-linear triangulated category $\mathcal{T}$.

Definition 4.2. An object $E \in \mathcal{T}$ is said to have a Serre dual $\mathrm{S} E$ if the contravariant functor $\operatorname{Hom}_{\mathcal{T}}(E,-)^{*}$ - the star stands for vector space dual-is represented by SE. If a Serre dual can be chosen functorially via an auto-equivalence $\mathrm{S}$, this functor $\mathrm{S}$ is said to be a Serre functor of $\mathcal{T}$.

Remark 4.3. The Calabi-Yau condition in definition 1.12(S3) for a $d$-spherlike object $E$ to be spherical demands that for all $F$, the composition pairing induce an isomorphism $\operatorname{Hom}_{D^{\mathrm{b}}}^{d}(E, F) \cong \operatorname{Hom}_{D^{\mathrm{b}}}(F, E)^{*}$ or, equivalently, that $E[-d]$ be a Serre dual for $E$; see also [ST01, lem. 2.15]. 
Let $E \in \mathcal{T}$ be a $d$-spherelike (but not necessarily spherical) object that has some Serre dual $\mathrm{S} E$. Since in particular $\operatorname{End}_{\mathcal{T}}^{d}(E)^{*}=\operatorname{Hom}_{\mathcal{T}}(E, E[-d])^{*} \cong \operatorname{Hom}_{\mathcal{T}}(E[-d], \mathrm{S} E)$, there is a morphism $x^{*}: E[-d] \rightarrow \mathrm{S} E$ dual to the non-trivial endomorphism $x \in \operatorname{End}_{\mathcal{T}}^{d}(E)$.

Definition 4.4. The asphericality of a spherelike object $E$ is $Q(E):=\operatorname{cone}\left(x^{*}\right)$. Its left complement ${ }^{\perp} Q(E):=\left\{X \in \mathcal{T} \mid \operatorname{Hom}_{\mathcal{T}}(X, Q(E))=0\right\}$ is a full triangulated subcategory of $\mathcal{T}$.

Theorem 4.5 (Hochenegger, Kalck, Ploog) [HKP16, Thm. 4.4, Appendix A]. The spherical subcategory $\operatorname{Sph}(E):={ }^{\perp} Q(E)$ of $E$ is the largest triangulated subcategory of $D^{\mathrm{b}}(\mathcal{T})$ in which $E$ is spherical.

Example 4.6. For $\mathfrak{g}$ a semisimple complex Lie algebra and $\lambda$ a regular weight (for instance, $\lambda=0$ ), the auto-equivalence $\mathrm{S}:=\mathbf{L} \mathrm{Sh}_{w_{0}}^{2}$ is a Serre functor of $D^{\mathrm{b}}\left(\mathcal{O}_{\lambda}\right)$ [MS08, Prop. 4.1].

Proposition 4.7. The 0 -spherelike module $P(s) \in D^{\mathrm{b}}\left(\mathcal{O}_{0, \mathfrak{s}_{3}}\right)$ has Serre dual $\mathrm{S} P(s) \cong P(s)^{\vee}$.

Proof. For this proof, we take the graded structure on $\mathcal{O}_{0}^{\mathbf{Z}}$ into account. Recall from definition 2.15 that $\Theta_{s}^{2} \cong \Theta_{s}\langle-1\rangle \oplus \Theta_{s}\langle 1\rangle$, and hence $\operatorname{Sh}_{s} \Theta_{s} \cong \Theta_{s}\langle-1\rangle$. Recall from (1.1) and (1.2) that for $w<w s$, the functor $\mathrm{Sh}_{s}$ maps standard factors $M(w)$ to $M(w s)$ and $M(w s)$ to $\left(\begin{array}{c}M(w s) \\ M(w) / M(w s)\end{array}\right)\langle-1\rangle$. Applying the factors of $\mathbf{S}=\mathbf{L} \mathrm{Sh}_{w_{0}}^{2}$ successively shows that $P(s)$ is mapped under $\mathrm{S}$ to

$$
\begin{aligned}
& P(s)=\Theta_{s} M(s) \\
& \stackrel{\mathrm{Sh}_{s}}{\longmapsto} P(s)\langle-1\rangle=\left(\begin{array}{c}
M(s) \\
M(e)
\end{array}\right)\langle-1\rangle \\
& \stackrel{\mathrm{Sh}_{t}}{\longmapsto}\left(\begin{array}{c}
M(t s) \\
M(t)
\end{array}\right)\langle-1\rangle \\
& \stackrel{\mathrm{Sh}_{s}}{\longmapsto}\left(\begin{array}{c}
M\left(w_{0}\right) \\
M(t s)
\end{array}\right)\langle-1\rangle=\Theta_{t} M(t s)\langle-1\rangle \\
& \stackrel{\mathrm{Sh}_{t}}{\longmapsto}\left(\begin{array}{c}
M\left(w_{0}\right) \\
M(t s)
\end{array}\right)\langle-2\rangle
\end{aligned}
$$

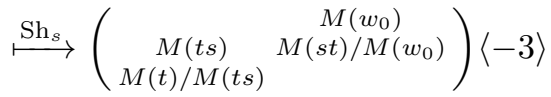

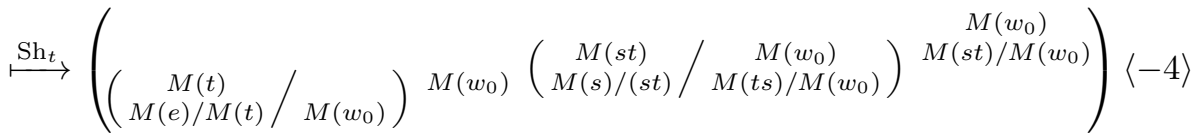

$$
\begin{aligned}
& =\left(\begin{array}{ccc} 
& L\left(w_{0}\right) \\
& L(s t) & L(t s) \\
L\left(w_{0}\right) & L(s) & L(t) \\
L(s t) & L(t s) & L(e) \\
L(s) &
\end{array}\right)\langle-4\rangle
\end{aligned}
$$

which is precisely the composition series of the dual module $P(s)^{\vee}$.

The space $\operatorname{Hom}\left(P(s), P(s)^{\vee}\right)=\operatorname{Hom}(P(s), \mathrm{S} P(s)) \cong \operatorname{End}(P(s))^{*}$ is spanned by the morphisms id ${ }^{*}$ and $x^{*}$ dual to id, $x \in \operatorname{End}_{\mathcal{O}}(P(s))$. According to the grading of $P(s)$ in $\mathcal{O}_{0}^{\mathbb{Z}}$, the maps id and $x$ respectively have degrees 0 and 2 , so $\mathrm{id}^{*}$ and $x^{*}$ are of degrees 0 and -2 . In 
terms of composition series of $P(s)$ and $P(s)^{\vee}$, the maps id* and $x^{*}$ are

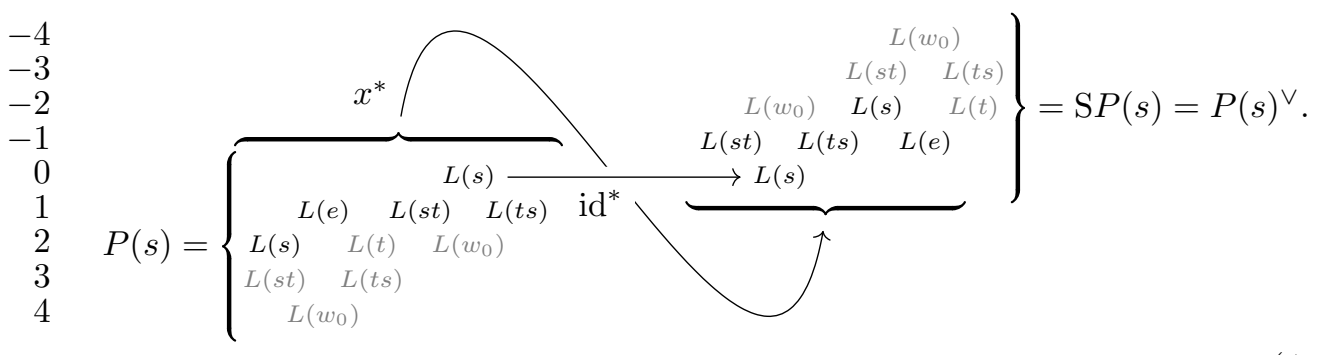

The grading of the modules is as indicated on the left, such that the two factors $L(s)$ connected by $\mathrm{id}^{*}$ are in degree zero and $x^{*}$ is a degree -2 -map indeed. The grey composition factors belong to the kernel and cokernel of $x^{*}$, respectively. Using this explicit description for $x^{*}$, we can prove the following:

Theorem 4.8. The inclusion $D^{\mathrm{b}}\left(\mathcal{O}_{0, \mathfrak{s l}_{2}}\right) \hookrightarrow D^{\mathrm{b}}\left(\mathcal{O}_{0, \mathfrak{s}_{3}}\right)$ from (4.1) factors through $\mathcal{S p h}(P(s))$.

Proof. From the composition series (4.2) of $P(s)^{\vee}$, we can derive that $P(s)^{\vee}$ has a projective resolution $\left\{P(s) \rightarrow P\left(w_{0}\right)\langle-2\rangle \rightarrow P\left(w_{0}\right)\langle-4\rangle\right.$. Expressiong the map $x^{*}$ from (4.3) in terms of the projective resolution of $P(s)^{\vee}$ yields that the asphericality $Q(P(s))=\operatorname{cone}\left(x^{*}\right)$ of $P(s)$ is the total complex

$$
Q \simeq\left\{\begin{array}{r}
P(s)\langle-2\rangle \\
\downarrow \\
P(s) \rightarrow P\left(w_{0}\right)\langle-2\rangle \rightarrow P\left(w_{0}\right)\langle-4\rangle
\end{array}\right\}
$$

with the canonical inclusions and with the bottom right $P\left(w_{0}\right)\langle-4\rangle$ is in homological degree 0 . We claim that

$$
\operatorname{Hom}_{D^{\mathrm{b}}\left(\mathcal{O}_{\left.0, \mathfrak{s}_{3}\right)}\right)}(P(w), Q) \begin{cases}=0 & \text { if } w \in\{e, s\}, \\ \neq 0 & \text { if } w \in\left\{t, s t, t s, w_{0}\right\} .\end{cases}
$$

Consider the composition series of the projective modules involved in (4.4). The $P\left(w_{0}\right)\langle-4\rangle$ in degree 0 has composition series

$$
P\left(w_{0}\right)\langle-4\rangle=\left(\begin{array}{cccc}
L\left(w_{0}\right) & \\
L(s) & L(t s) & \\
L(t s) & L\left(w_{0}\right) & L\left(w_{0}\right) & L(t) \\
L(s t) & L(t s) & L(e) & L(s t) \\
L\left(w_{0}\right) & L(s) & L(t) & L(t s) \\
& L(s t) & L(t s) & \\
& L\left(w_{0}\right) &
\end{array}\right)\langle-4\rangle ;
$$

the gray factors are a composition series of the image of

$$
\left(P(s)\langle-2\rangle \oplus P\left(w_{0}\right)\langle-2\rangle \rightarrow P\left(w_{0}\right)\langle-4\rangle,\right.
$$

which is the last non-trivial map of the total complex (4.4). Every map $P(e)\langle-\rangle \rightarrow P\left(w_{0}\right)\langle-4\rangle$ and $P(s)\langle-\rangle \rightarrow P\left(w_{0}\right)\langle-4\rangle$ factors through $P(s)\langle-2\rangle \oplus P\left(w_{0}\right)\langle-2\rangle$ and hence is nullhomotopic. This shows that $\operatorname{Hom}_{D^{\mathrm{b}}\left(\mathcal{O}\left(\mathfrak{s}_{3}\right)\right)}(P(w), Q)=0$ if $w \in\{e, s\}$. 
For $w \in\left\{t, s t, t s, w_{0}\right\}$ on the other hand, the black composition factors $L(w)$ in $(4.5)$ generate images of non-zero morphisms $P(w)\langle-\rangle \rightarrow P\left(w_{0}\right)\langle-2\rangle$ that cannot be factored through $P(s)\langle-2\rangle \oplus P\left(w_{0}\right)\langle-2\rangle$ and thus are not null-homotopic. These morphisms therefore represent non-trivial morphisms $P(w)\langle-\rangle \rightarrow Q$ in $D^{\mathrm{b}}\left(\mathcal{O}_{0, \mathfrak{s l}_{3}}\right)$ and thus show that

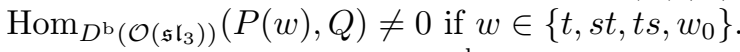

We see that $\mathcal{S p h}(P(s))$ of $D^{\mathrm{b}}\left(\mathcal{O}_{0, \mathfrak{s}_{3}}\right)$ contains the triangulated subcategory generated by $P(s)$ and $P(e)$, which proves the claim.

4.2 Maximal parabolic subalgebras We shall now address parabolic subalgebras $\mathfrak{p}$ of $\mathfrak{s l}_{3}$ (section 2.5) as another remedy for the failure of the Calabi-Yau property of $P(s)$ in $\mathcal{O}_{0, \mathfrak{s l}_{3}}$. Consider the category $\mathcal{O}_{0}^{\mathfrak{p}}$ corresponding to the parabolic subgroup $W_{\mathfrak{p}}=\langle t\rangle \cong S_{1} \times S_{2}$ of $W=S_{3}=\langle s, t\rangle$. The minimal-length representatives of cosets in $W / W_{\mathfrak{p}}$ are $W^{\mathfrak{p}}=\{e, s, s t\}$. There is an equivalence $\mathcal{O}_{0}^{\mathfrak{p}} \simeq A_{\mathfrak{p}}$-Mod for the path algebra quotient

$$
A_{\mathfrak{p}}:=A_{\mathfrak{s l}_{3}} /\left(\varepsilon_{t}, \varepsilon_{t s}, \varepsilon_{w_{0}}\right)=\mathbf{C}[e \rightleftarrows s \rightleftarrows s t] /\left(\begin{array}{c}
e \leftarrow s \leftarrow e=0, \quad e \leftarrow s \leftarrow s t=0, \\
s t \leftarrow s \leftarrow e=0, \quad s \leftarrow e \leftarrow s=s \leftarrow s t \leftarrow s
\end{array}\right)
$$

of the path algebra $A_{\mathfrak{s l}_{3}}$. The parabolic Verma modules and projectives have the following composition series, whose factors now are simple $A_{\mathfrak{p}}$-modules:

\begin{tabular}{|c|c|c|c|c|c|}
\hline$M^{\mathfrak{p}}(e)$ & $M^{\mathfrak{p}}(s)$ & $M^{\mathfrak{p}}(s t)$ & $P^{\mathfrak{p}}(e)$ & $P^{\mathfrak{p}}(s)$ & $P^{\mathfrak{p}}(s t)$ \\
\hline $\begin{array}{l}L(e) \\
L(s)\end{array}$ & $\begin{array}{l}L(s) \\
L(s t)\end{array}$ & $L(s t)$ & $M(e)$ & $\begin{array}{c}M(s) \\
M(e) \\
=L(s t) \quad L(e) \\
L(s)\end{array}$ & $\begin{array}{cc}M(s t) & L(s t) \\
M(s) & L(s) \\
& L(s t)\end{array}$ \\
\hline
\end{tabular}

According to remark 2.22, the defining short exact sequences (1.1) of $\Theta_{s}$ and $\mathbf{L} \mathrm{Sh}_{s}$ restrict to sequences $(2.7)$ in $\mathcal{O}_{0}^{\mathfrak{p}}$ so the indecomposable projective modules have the following images under $\Theta_{s}$ and $\mathbf{L} \mathrm{Sh}_{s}$ :

\begin{tabular}{|c|c|c|c|c|}
\hline$M$ & $\Theta_{s} M$ & $\mathbf{L ~ S h}{ }_{s} M$ & $\Theta_{t} M$ & $\mathbf{L ~ S h}{ }_{t} M$ \\
\hline$P^{\mathfrak{p}}(e)$ & $P^{\mathfrak{p}}(s)$ & $\left\{P^{\mathfrak{p}}(e) \rightarrow P^{\mathfrak{p}}(s)\right\}$ & 0 & $P^{\mathfrak{p}}(e)[-1]$ \\
\hline$P^{\mathfrak{p}}(s)$ & $P^{\mathfrak{p}}(s) \oplus P^{\mathfrak{p}}(s)$ & $P^{\mathfrak{p}}(s)$ & $P^{\mathfrak{p}}(s t)$ & $\left\{P^{\mathfrak{p}}(s) \rightarrow P^{\mathfrak{p}}(s t)\right\}$ \\
\hline$P^{\mathfrak{p}}(s t)$ & $P^{\mathfrak{p}}(s)$ & $\left\{P^{\mathfrak{p}}(s t) \rightarrow P_{0}^{\mathfrak{p}}(s)\right\}$ & $P^{\mathfrak{p}}(s t)^{\oplus 2}$ & $P^{\mathfrak{p}}(s t)$ \\
\hline
\end{tabular}

Remark 4.9. A module $M$ is $\mathrm{Sh}_{w}$-acyclic if and only if $\mathbf{L} \mathrm{Sh}_{w}$ is quasi-isomorphic to a complex concentrated in degree zero. The results form (4.8) therefore are examples for Caveat 2.23: the objects $P^{\mathfrak{p}}(-)$, albeit projective in the category $\mathcal{O}_{0}^{\mathfrak{p}}$, are not $\mathrm{Sh}_{s}$-acyclic and hence in particular not projective in $\mathcal{O}_{0}$.

Lemma 4.10. The set $\left\{P^{\mathfrak{p}}(s), P^{\mathfrak{p}}(s t)\right\}$ is an $\mathrm{A}_{2}$-collection of 0 -spherical objects in $D^{\mathrm{b}}\left(\mathcal{O}_{0}^{\mathfrak{p}}\right)$.

Proof. From the composition series in (4.7), we see that $P^{\mathfrak{p}}(s)$ and $P^{\mathfrak{p}}(s t)$ have endomorphism algebras isomorphic to $\mathbf{C}[x] /\left(x^{2}\right)$, with the non-trivial endomorphism $x: P^{\mathfrak{p}}(w) \rightarrow L(w) \hookrightarrow$ $P^{\mathfrak{p}}(w)$ for $w \in\{s, s t\}$. All Hom-spaces in the following are one-dimensional, and we see that for the indecomposable projectives, the composition pairings

$$
\begin{aligned}
& \operatorname{Hom}_{\mathcal{O}}\left(P^{\mathfrak{p}}(e), P^{\mathfrak{p}}(s)\right) \otimes \operatorname{Hom}_{\mathcal{O}}\left(P^{\mathfrak{p}}(s), P^{\mathfrak{p}}(e)\right) \longrightarrow \operatorname{End}\left(P^{\mathfrak{p}}(s)\right) /\langle\mathrm{id}\rangle
\end{aligned}
$$

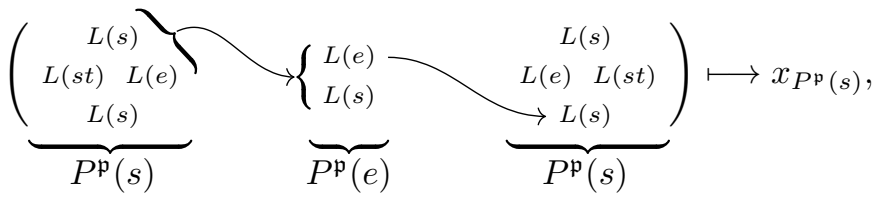




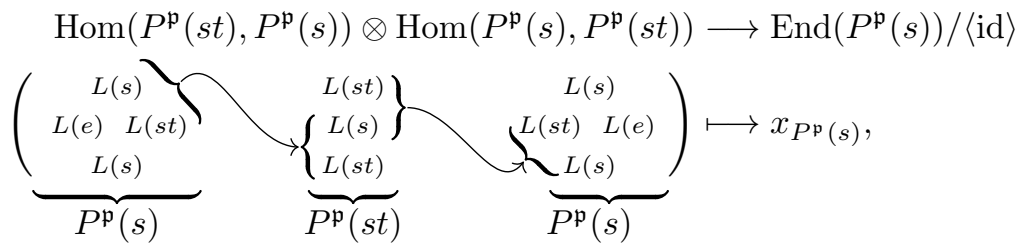

$$
\operatorname{Hom}_{\mathcal{O}}\left(P^{\mathfrak{p}}(s), P^{\mathfrak{p}}(s t)\right) \otimes \operatorname{Hom}_{\mathcal{O}}\left(P^{\mathfrak{p}}(s), P^{\mathfrak{p}}(s t)\right) \longrightarrow \operatorname{End}\left(P^{\mathfrak{p}}(p s)\right) /\langle\mathrm{id}\rangle
$$

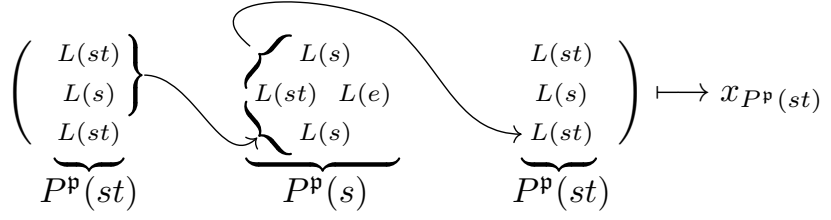

are non-degenerate and that $\operatorname{Hom}_{\mathcal{O}}\left(P^{\mathfrak{p}}(e), P^{\mathfrak{p}}(e)(s)\right)=0=\operatorname{Hom}_{\mathcal{O}}\left(P^{\mathfrak{p}}(s t), P^{\mathfrak{p}}(e)(e)\right)$. Hence, $P^{\mathfrak{p}}(s)$ and $P^{\mathfrak{p}}(s t)$ are 0 -spherical objects in $D^{\mathrm{b}}\left(\mathcal{O}_{0}^{\mathfrak{p}}\right)$. In particular, $\operatorname{dim} \operatorname{Hom}\left(P^{\mathfrak{p}}(s), P^{\mathfrak{p}}(s t)\right)=$ $1=\operatorname{Hom}\left(P^{\mathfrak{p}}(s t), P^{\mathfrak{p}}(s)\right)$ as required; so the set $\left\{P^{\mathfrak{p}}(s), P^{\mathfrak{p}}(s t)\right\}$ is an $\mathrm{A}_{2}$-configuration.

With the dimensions of the hom-spaces in (4.9-4.10), it follows immediately from definition 1.13 that the indecomposable projective modules under $T_{P(s)}^{\prime}$ and $T_{P(s t)}^{\prime}$ are

$$
\begin{aligned}
T_{P^{\mathfrak{p}}(s)}^{\prime}: P^{\mathfrak{p}}(e) & \mapsto\left\{P^{\mathfrak{p}}(e) \rightarrow P^{\mathfrak{p}}(s)\right\} & T_{P^{\mathfrak{p}}(s t)}^{\prime}: P^{\mathfrak{p}}(e) & \mapsto P^{\mathfrak{p}}(e) \\
P^{\mathfrak{p}}(s) & \mapsto P^{\mathfrak{p}}(s)[1] & P^{\mathfrak{p}}(s) & \mapsto\left\{P^{\mathfrak{p}}(s) \rightarrow P^{\mathfrak{p}}(s t)\right\} \\
P^{\mathfrak{p}}(s t) & \mapsto\left\{P^{\mathfrak{p}}(s t) \rightarrow P^{\mathfrak{p}}(s)\right\} & P^{\mathfrak{p}}(s t) & \mapsto
\end{aligned}
$$

Proposition 4.11. For $\mathfrak{p}$ as above, there are natural isomorphisms $T_{P^{\mathfrak{p}}(s)}^{\prime} \cong \mathbf{L} \mathrm{Sh}_{s}[1]$ and $T_{P^{\mathfrak{p}}(s t)}^{\prime} \cong \mathbf{L} \mathrm{Sh}_{t}[1]$ of autoequivalences of $\mathcal{O}_{0}^{\mathfrak{p}}$.

Proof. We see from (4.8) and (4.12) that $T_{P^{\mathfrak{p}}(s)}^{\prime} P^{\mathfrak{p}}(w) \simeq \mathbf{L} \operatorname{Sh}_{s} P^{\mathfrak{p}}(w)[1]$ and $T_{P^{\mathfrak{p}}(s t)}^{\prime} P^{\mathfrak{p}}(w) \simeq$ $\mathbf{L} \operatorname{Sh}_{t} P^{\mathfrak{p}}(w)[1]$ for all $w \in W^{\mathfrak{p}}$. It remains to show that the shuffling and spherical twist functors also map elements of $\operatorname{Hom}_{\mathcal{O}^{\mathfrak{p}}}\left(P^{\mathfrak{p}}(v), P^{\mathfrak{p}}(w)\right.$ ) (for $v, w \in W^{\mathfrak{p}}$ ) to isomorphic maps. This will be carried out analogously to theorem 3.6.

The proof for $\Theta_{t} \cong T_{P^{\mathfrak{p}}(s t)}^{\prime}$ is done, mutatis mutandis, the same way as for $\Theta_{s} \cong T_{P^{\mathfrak{p}}(s)}^{\prime}$; we thus only show the latter. To that end, we show that the $A_{\mathfrak{p}}-A_{\mathfrak{p}}$-bimodules $M_{\Xi_{P}^{\prime}{ }^{\prime}(s)}$ and $M_{\Theta_{s}}$, for which the functors $-\otimes M_{\Xi_{P \mathfrak{p}(s)}^{\prime}}$ and $-\otimes M_{\Theta_{s}}$ respectively correspond to $\Xi_{P^{\mathfrak{p}}(s)}^{\prime}$ and $\Theta_{s}$ under $\mathcal{O}_{0}^{\mathfrak{p}} \simeq \operatorname{Mod}-A_{\mathfrak{p}}$, are isomorphic.

Since as an $A_{\mathfrak{p}}$-module, $P^{\mathfrak{p}}(s)=\varepsilon_{s} A_{\mathfrak{p}}$ has an explicit finite basis by paths of the quiver (4.6) ending in the vertex $s$, the module $M_{\Xi_{P \mathfrak{p}(s)}^{\prime}}=P^{\mathfrak{p}}(s)^{*} \otimes_{\mathbf{C}} P^{\mathfrak{p}}(s)$ - the star stands for vector space dual - has a $\mathbf{C}$-basis given by the sixteen pairwise tensor products in the schematic

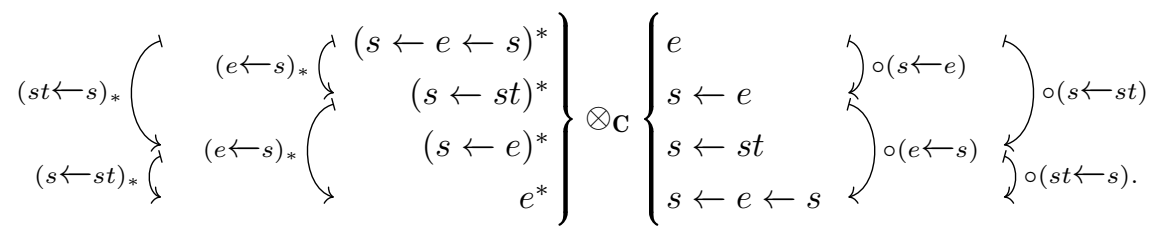

The right $A_{\mathfrak{p}}$-action is given by precomposition of paths; under the left $A_{\mathfrak{p}}$-action, a $\gamma \in A_{\mathfrak{p}}$ acts on a $\lambda \in P^{\mathfrak{p}}(s)^{*}$ by $\gamma_{*}(\lambda): v \mapsto \lambda(v \circ \gamma)$. The $A_{\mathfrak{p}}-A_{\mathfrak{p}^{-}}$-bimodule action on $M_{\Xi_{\Xi^{\prime}(s)}^{\prime}}$ hence is such that the generating paths of $A_{\mathfrak{p}}$ act from the left and right as indicated. 
To construct the module $M_{\Theta_{s}}$, we consider the endomorphism algebra $\operatorname{End}_{\mathcal{O}}\left(P^{\mathfrak{p}}\right)$ of $P^{\mathfrak{p}}=P^{\mathfrak{p}}(e) \oplus P^{\mathfrak{p}}(s) \oplus P^{\mathfrak{p}}(s t)$, which is generated by the elements

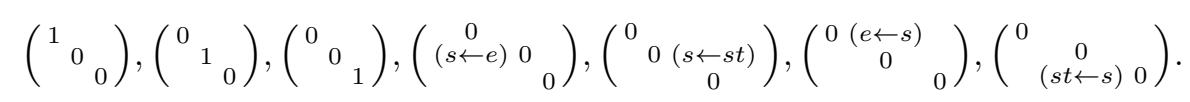

Since according to $(4.8)$ we have $\Theta_{s} P^{\mathfrak{p}}=P^{\mathfrak{p}}(s)^{\oplus 4}$, we endow these $P^{\mathfrak{p}}(s)$ 's with indices to make them distinguishable, identifying $P^{\mathfrak{p}}(s)_{1}$ with $\Theta_{s} P^{\mathfrak{p}}(e), P^{\mathfrak{p}}(s)_{2} \oplus P^{\mathfrak{p}}(s)_{3}$ with $\Theta_{s} P^{\mathfrak{p}}(s)$ and $P^{\mathfrak{p}}(s)_{4}$ with $\Theta_{s} P^{\mathfrak{p}}(s t)$. A diagram chase of morphisms through the relevant naturality diagrams shows that $\Theta_{s}$ respectively maps the above generators of $\operatorname{End}_{\mathcal{O}}\left(P^{\mathfrak{p}}\right)$ from (4.14) to the endomorphisms

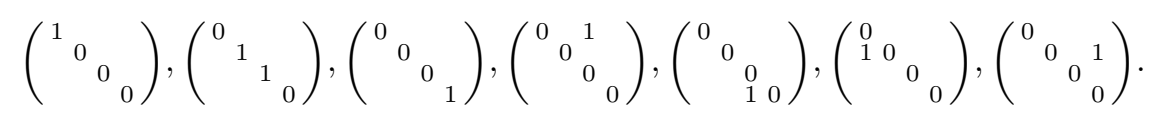

of $\Theta_{s} P^{\mathfrak{p}}=P^{\mathfrak{p}}(s)^{\oplus 4}$. Somewhat more suggestively, we may write these morphisms $f$ as arrows connecting the two $P^{\mathfrak{p}}(-)$ 's on which they have non-zero kernel or cokernel. $\Theta_{s}$ then maps the last four of the generators $f$ from the table, which we depict by the following morphisms on the left, to the respective solid or dashed morphism on the right:

$$
\begin{aligned}
& P^{\mathfrak{p}}=P(e) \oplus P(s) \oplus P(s t) \\
& P(e) \oplus P(s) \oplus P(s t) \\
& P(e) \oplus P(s) \stackrel{k^{\prime}-}{\oplus} P(s t)
\end{aligned}
$$

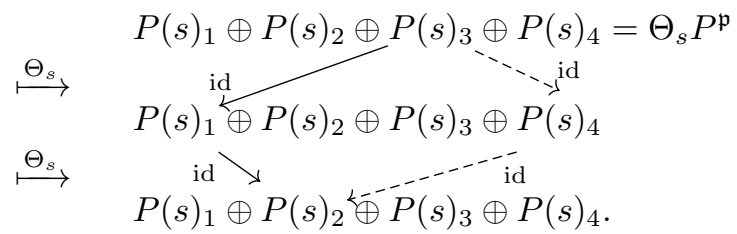

A vector space basis of $M_{\Theta_{s}}=\operatorname{Hom}\left(P^{\mathfrak{p}}, \Theta_{s} P^{\mathfrak{p}}\right)$ is given by morphisms sending $P^{\mathfrak{p}}$ to one of its summands $P^{\mathfrak{p}}(w)$ (where $w \in\{e, s, s t\}$ ) and further to a summand $P^{\mathfrak{p}}(s)_{i}$ of $\Theta_{s} P^{\mathfrak{p}}$. In other words, this basis consists of the 16 possible ways to map a summand $P^{\mathfrak{p}}(w)$ of $P^{\mathfrak{p}}$ on the right to the $P^{\mathfrak{p}}(s)$ in the middle and embed this into $\Theta_{s} P^{\mathfrak{p}}$ as one of the summands on the left:

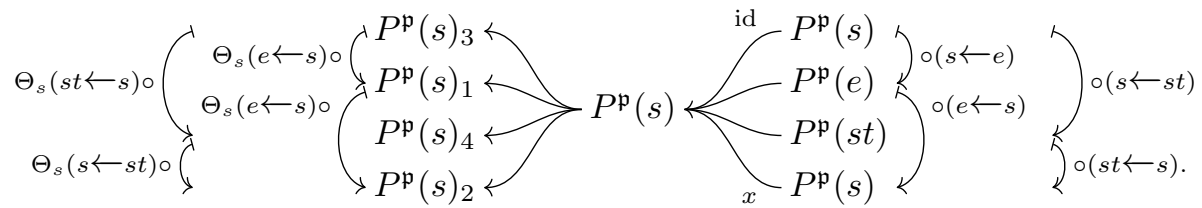

To understand the $A^{\mathfrak{p}}-A^{\mathfrak{p}}$-bimodule action on $M_{\Theta_{s}}$, recall from corollary 3.8 that $\phi, \psi \in A^{\mathfrak{p}}$ act on $m \in M_{\Theta_{s}}$ by $\phi . m . \psi=\Theta_{s}(\phi) \circ m \circ \psi$, with the images $\Theta_{s}(\phi)$ from (4.15) and (4.16). On the vector space basis of $M_{\Theta_{s}}$ from (4.17), the generating paths of the algebra $A^{\mathfrak{p}}$ therefore act from the left and right as indicated.

Comparing (4.13) and (4.17) shows that the obvious isomorphism $M_{\Xi_{P^{p}(s)}^{\prime}} \cong M_{\Theta_{s}}$ of vector spaces is an isomorphism of $A^{\mathfrak{p}}-A^{\mathfrak{p}}$-bimodules. It follows that $T_{P^{\mathfrak{p}}(s)}^{\prime} \simeq\left\{\mathrm{id} \Rightarrow-\otimes M_{\Xi_{P}^{\prime}(s)}\right\}$ and $\mathrm{Sh}_{s}=\{\mathrm{id} \Rightarrow-\} \cong M_{\Theta_{s}}$ are naturally isomorphic functors.

Parabolic subalgebras of $\mathfrak{s l}_{\boldsymbol{n}}$ We now transfer results for $\mathcal{O}_{0}^{\mathfrak{p}}$ from $\mathfrak{s l}_{3}$ to $\mathfrak{s l}_{n}$. Consider the parabolic subalgebra $\mathfrak{p}$ of $\mathfrak{s l}_{n}$ corresponding to the subgroup $W_{\mathfrak{p}}=\left\langle s_{2}, \ldots, s_{n-1}\right\rangle=S_{1} \times S_{n-1}$ of $S_{n}=\left\langle s_{1}, \ldots, s_{n-1}\right\rangle$. We let $\sigma_{0}:=e$ and $\sigma_{i}:=s_{1} \cdots s_{i}$ for $i \geq 1$; the minimal length coset representatives then are $W^{\mathfrak{p}}=\left\{\sigma_{0}, \ldots, \sigma_{n-1}\right\}$. 
Table 4.1: Composition series of Verma modules and indecomposable projectives indexed by the $\sigma_{i} \in W^{\mathfrak{p}}$.

\begin{tabular}{c|ccccc}
\hline & $\sigma_{0}$ & $\sigma_{1}$ & $\sigma_{2}$ & $\cdots$ & $\sigma_{n-1}$ \\
\hline$M^{\mathfrak{p}}(-)$ & $L(e)$ & $L\left(\sigma_{1}\right)$ & $L\left(\sigma_{2}\right)$ & $\ldots$ & $L\left(\sigma_{n-1}\right)$ \\
& $L\left(s_{1}\right)$ & $L\left(\sigma_{2}\right)$ & $L\left(\sigma_{3}\right)$ & & \\
& & $L\left(\sigma_{1}\right)$ & $L\left(\sigma_{2}\right)$ & & $L\left(\sigma_{n-1}\right)$ \\
$P^{\mathfrak{p}}(-)$ & $L(e)$ & $L(e) L\left(\sigma_{2}\right)$ & $L\left(s_{1}\right) L\left(\sigma_{3}\right)$ & $\cdots$ & $L\left(\sigma_{n-2}\right)$ \\
& $L\left(s_{1}\right)$ & $L\left(\sigma_{1}\right)$ & $L\left(\sigma_{2}\right)$ & & $L\left(\sigma_{n-1}\right)$ \\
\hline
\end{tabular}

Table 4.2: Images of Verma modules and indecomposable projectives under translation functors.

\begin{tabular}{c|cccc}
\hline$M$ & $\Theta_{s_{1}} M$ & $\Theta_{s_{2}} M$ & $\Theta_{s_{3}} M$ & $\cdots$ \\
\hline$M^{\mathfrak{p}}\left(\sigma_{0}\right)$ & $P^{\mathfrak{p}}\left(\sigma_{1}\right)$ & & & \\
$M^{\mathfrak{p}}\left(\sigma_{1}\right)$ & $P^{\mathfrak{p}}\left(\sigma_{1}\right)$ & $P^{\mathfrak{p}}\left(\sigma_{2}\right)$ & & \\
$M^{\mathfrak{p}}\left(\sigma_{2}\right)$ & $P^{\mathfrak{p}}\left(\sigma_{1}\right)$ & $P^{\mathfrak{p}}\left(\sigma_{2}\right)$ & $P^{\mathfrak{p}}\left(\sigma_{3}\right)$ & \\
$M^{\mathfrak{p}}\left(\sigma_{3}\right)$ & & $P^{\mathfrak{p}}\left(\sigma_{2}\right)$ & $P^{\mathfrak{p}}\left(\sigma_{3}\right)$ & \\
$M^{\mathfrak{p}}\left(\sigma_{4}\right)$ & & & $P^{\mathfrak{p}}\left(\sigma_{3}\right)$ & $\ddots$ \\
\hline
\end{tabular}

\begin{tabular}{c|cccc}
\hline$M$ & $\Theta_{s_{1}} M$ & $\Theta_{s_{2}} M$ & $\Theta_{s_{3}} M$ & $\cdots$ \\
\hline$P^{\mathfrak{p}}\left(\sigma_{0}\right)$ & $P^{\mathfrak{p}}\left(\sigma_{1}\right)$ & & & \\
$P^{\mathfrak{p}}\left(\sigma_{1}\right)$ & $P^{\mathfrak{p}}\left(\sigma_{1}\right)^{2}$ & $P^{\mathfrak{p}}\left(\sigma_{2}\right)$ & & \\
$P^{\mathfrak{p}}\left(\sigma_{2}\right)$ & $P^{\mathfrak{p}}\left(\sigma_{1}\right)$ & $P^{\mathfrak{p}}\left(\sigma_{2}\right)^{2}$ & $P^{\mathfrak{p}}\left(\sigma_{3}\right)$ & \\
$P^{\mathfrak{p}}\left(\sigma_{3}\right)$ & & $P^{\mathfrak{p}}\left(\sigma_{2}\right)$ & $P^{\mathfrak{p}}\left(\sigma_{3}\right)^{2}$ & \\
$P^{\mathfrak{p}}\left(\sigma_{4}\right)$ & & & $P^{\mathfrak{p}}\left(\sigma_{3}\right)$ & $\ddots$ \\
\hline
\end{tabular}

Lemma 4.12. The category $\mathcal{O}_{0}^{\mathfrak{p}}\left(\mathfrak{s l}_{n}\right)$ is equivalent to $\operatorname{Mod}-A^{\mathfrak{p}}\left(\mathfrak{s l}_{n}\right)$ for

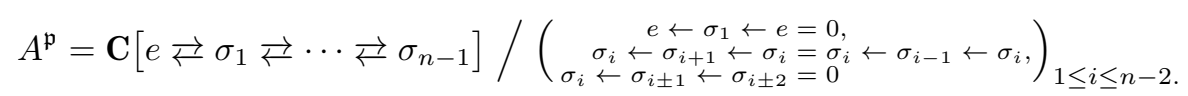

We shall denote this quiver by $Q^{\mathfrak{p}}$.

Proof. We compute the composition series of Verma modules and indecomposable projectives in $\mathcal{O}_{0}^{\mathfrak{p}}$ using the generalised Kazhdan-Lusztig theorem [Irv90, Cor. 7.1.3; CC87, Thm. 1.3]. For parabolic subgroups of the form $W_{\mathfrak{p}}=S_{k} \times S_{n-k} \leq S_{n}$ there is a graphical calculus for computing parabolic Kazhdan-Lusztig polynomials [BS11a, §5; LS13]. The composition series thus obtained are listed in table 4.1. These composition series show that the unique (up to scalar) morphisms $P^{\mathfrak{p}}\left(\sigma_{i}\right) \rightarrow P^{\mathfrak{p}}\left(\sigma_{i \pm 1}\right)$ are irreducible, that they generate End $_{\mathcal{O}^{\mathfrak{p}}} \bigoplus_{i=0}^{n-1} P\left(\sigma_{i}\right)$, and that their relations generate precisely the ideal quotiented out in the statement of the lemma.

Every Verma module $M^{\mathfrak{p}}\left(\sigma_{i}\right)$ fits uniquely into a short exact sequence

$$
M^{\mathfrak{p}}\left(\sigma_{i}\right) \hookrightarrow P^{\mathfrak{p}}\left(\sigma_{i+1}\right) \rightarrow M^{\mathfrak{p}}\left(\sigma_{i+1}\right) ;
$$

in particular, $\Theta_{s_{i}} M^{\mathfrak{p}}\left(\sigma_{i}\right)=P^{\mathfrak{p}}\left(\sigma_{i}\right)$ is always projective, which is not true in the non-parabolic category $\mathcal{O}_{0}$. From these sequences we obtain the images of the indecomposable projective and Verma modules $M$ under translation and shuffling functors listed in tables 4.2 to 4.4 (two-term complexes are understood to have the right entry in degree zero).

Lemma 4.13. $\left\{P^{\mathfrak{p}}\left(\sigma_{1}\right), \ldots, P^{\mathfrak{p}}\left(\sigma_{n-1}\right)\right\}$ is an $\mathrm{A}_{n-2}$-configuration of 0 -spherical objects.

Proof. The composition series from table 4.1 exhibit that $\operatorname{Hom}_{\mathcal{O}_{0}^{\mathfrak{p}}}\left(P^{\mathfrak{p}}\left(\sigma_{i}\right), P^{\mathfrak{p}}\left(\sigma_{i}\right)\right) \cong \mathbf{C}[x] /\left(x^{2}\right)$ for all $1 \leq i \leq n-1$, that the nontrivial endomorphism $x$ is the degree 2-map

$$
x: P^{\mathfrak{p}}\left(\sigma_{i}\right) \rightarrow L^{\mathfrak{p}}\left(\sigma_{i}\right) \hookrightarrow P^{\mathfrak{p}}\left(\sigma_{i}\right)
$$


Table 4.3: Images of Verma modules under derived shuffling functors.

\begin{tabular}{c|cccc}
\hline \multicolumn{1}{c}{$\mid \mathbf{L ~ S h}_{s_{1}} M$} & \multicolumn{1}{c}{$\mathbf{L ~ S h}_{s_{2}} M$} & \multicolumn{1}{c}{$\mathbf{L ~ S h}_{s_{3}} M$} & $\cdots$ \\
\hline$M^{\mathfrak{p}}\left(\sigma_{0}\right)$ & & $M^{\mathfrak{p}}\left(\sigma_{1}\right)$ & $M^{\mathfrak{p}}\left(\sigma_{0}\right)[-1]$ & $M^{\mathfrak{p}}\left(\sigma_{0}\right)[-1]$ \\
$M^{\mathfrak{p}}\left(\sigma_{1}\right)$ & $\left.\left\{M^{\mathfrak{p}}\left(\sigma_{1}\right) \longrightarrow P^{\mathfrak{p}}\left(\sigma_{1}\right)\right)\right\}$ & $M^{\mathfrak{p}}\left(\sigma_{2}\right)$ & $M^{\mathfrak{p}}\left(\sigma_{1}\right)[-1]$ \\
$M^{\mathfrak{p}}\left(\sigma_{2}\right)$ & $M^{\mathfrak{p}}\left(\sigma_{2}\right)[-1]$ & $\left\{M^{\mathfrak{p}}\left(\sigma_{2}\right) \longrightarrow P^{\mathfrak{p}}\left(\sigma_{2}\right)\right\}$ & $M^{\mathfrak{p}}\left(\sigma_{3}\right)$ & \\
$M^{\mathfrak{p}}\left(\sigma_{3}\right)$ & $M^{\mathfrak{p}}\left(\sigma_{3}\right)[-1]$ & $M^{\mathfrak{p}}\left(\sigma_{3}\right)[-1]$ & $\left\{M^{\mathfrak{p}}\left(\sigma_{3}\right) \rightarrow P^{\mathfrak{p}}\left(\sigma_{3}\right)\right\}$ & \\
$M^{\mathfrak{p}}\left(\sigma_{4}\right)$ & $M^{\mathfrak{p}}\left(\sigma_{4}\right)[-1]$ & $M^{\mathfrak{p}}\left(\sigma_{4}\right)[-1]$ & $M^{\mathfrak{p}}\left(\sigma_{4}\right)$ & \\
$M^{\mathfrak{p}}\left(\sigma_{5}\right)$ & $M^{\mathfrak{p}}\left(\sigma_{5}\right)[-1]$ & $M^{\mathfrak{p}}\left(\sigma_{5}\right)[-1]$ & $M^{\mathfrak{p}}\left(\sigma_{5}\right)$ & $\ddots$ \\
\hline
\end{tabular}

Table 4.4: Images of indecomposable projectives under derived shuffling functors.

\begin{tabular}{|c|c|c|c|c|}
\hline$M$ & $\mathbf{L} \mathrm{Sh}_{s_{1}} M$ & $\mathbf{L} \mathrm{Sh}_{s_{2}} M$ & $\mathbf{L} \mathrm{Sh}_{s_{3}} M$ & $\ldots$ \\
\hline$P^{\mathfrak{p}}\left(\sigma_{0}\right)$ & $M^{\mathfrak{p}}\left(\sigma_{1}\right)$ & $P^{\mathfrak{p}}\left(\sigma_{0}\right)[-1]$ & $P^{\mathfrak{p}}\left(\sigma_{0}\right)[-1]$ & \\
\hline$P^{\mathfrak{p}}\left(\sigma_{1}\right)$ & $\left.P^{\mathfrak{p}}\left(\sigma_{1}\right)\right)$ & $\left\{P^{\mathfrak{p}}\left(\sigma_{1}\right) \longrightarrow P^{\mathfrak{p}}\left(\sigma_{2}\right)\right\}$ & $P^{\mathfrak{p}}\left(\sigma_{1}\right)[-1]$ & \\
\hline$P^{\mathfrak{p}}\left(\sigma_{2}\right)$ & $\left.\left\{P^{\mathfrak{p}}\left(\sigma_{2}\right) \longrightarrow P^{\mathfrak{p}}\left(\sigma_{1}\right)\right)\right\}$ & $P^{\mathfrak{p}}\left(\sigma_{2}\right)$ & $\left\{P^{\mathfrak{p}}\left(\sigma_{2}\right) \longrightarrow P^{\mathfrak{p}}\left(\sigma_{3}\right)\right\}$ & \\
\hline$P^{\mathfrak{p}}\left(\sigma_{3}\right)$ & $P^{\mathfrak{p}}\left(\sigma_{3}\right)[-1]$ & $\left\{P^{\mathfrak{p}}\left(\sigma_{3}\right) \longrightarrow P^{\mathfrak{p}}\left(\sigma_{2}\right)\right\}$ & $P^{\mathfrak{p}}\left(\sigma_{3}\right)$ & \\
\hline$P^{\mathfrak{p}}\left(\sigma_{4}\right)$ & $P^{\mathfrak{p}}\left(\sigma_{4}\right)[-1]$ & $P^{\mathfrak{p}}\left(\sigma_{4}\right)[-1]$ & $\left\{P^{\mathfrak{p}}\left(\sigma_{4}\right) \longrightarrow P^{\mathfrak{p}}\left(\sigma_{2}\right)\right\}$ & \\
\hline$P^{\mathfrak{p}}\left(\sigma_{5}\right)$ & $P^{\mathfrak{p}}\left(\sigma_{5}\right)[-1]$ & $P^{\mathfrak{p}}\left(\sigma_{5}\right)[-1]$ & $P^{\mathfrak{p}}\left(\sigma_{5}\right)[-1]$ & \\
\hline
\end{tabular}

and that

$$
\operatorname{dim} \operatorname{Hom}_{\mathcal{O}_{0}^{\mathfrak{p}}}\left(P^{\mathfrak{p}}\left(\sigma_{j}\right), P^{\mathfrak{p}}\left(\sigma_{i}\right)\right)=\left\{\begin{array}{l}
2 \text { if } i=j \\
1 \text { if }|i-j|=1 \\
0 \text { otherwise. }
\end{array}\right.
$$

It is sufficient to check non-degeneracy of the composition pairing

$$
\operatorname{Hom}_{\mathcal{O}_{0}^{\mathfrak{p}}}\left(-, P^{\mathfrak{p}}\left(\sigma_{i}\right)\right) \otimes \operatorname{Hom}_{\mathcal{O}_{0}^{\mathfrak{p}}}\left(P^{\mathfrak{p}}\left(\sigma_{i}\right),-\right) \rightarrow\left\langle x_{P^{\mathfrak{p}}\left(\sigma_{i}\right)}\right\rangle
$$

only for the indecomposable projectives $P^{\mathfrak{p}}\left(\sigma_{i \pm 1}\right)$ that are connected to $P^{\mathfrak{p}}\left(\sigma_{i}\right)$ by an arrow in $Q^{\mathfrak{p}}$ because for $j \notin\{i-1, i, i+1\}$, the composition pairing

$$
\underbrace{\operatorname{Hom}_{\mathcal{O}_{0}^{\mathfrak{p}}}\left(P^{\mathfrak{p}}\left(\sigma_{j}\right), P^{\mathfrak{p}}\left(\sigma_{i}\right)\right)}_{0} \otimes \underbrace{\operatorname{Hom}_{\mathcal{O}_{0}^{\mathfrak{p}}}\left(P^{\mathfrak{p}}\left(\sigma_{i}\right), P^{\mathfrak{p}}\left(\sigma_{j}\right)\right)}_{0} \rightarrow\left\langle x_{P^{\mathfrak{p}}\left(\sigma_{i}\right)}\right\rangle
$$

is non-degenerate trivially.

For $P^{\mathfrak{p}}\left(\sigma_{i \pm 1}\right)$, we see, analoguously to (4.9-4.11), that the composition of the only (up to scalars) non-zero morphisms to and from $P^{\mathfrak{p}}\left(\sigma_{i}\right)$, which are written down in terms of

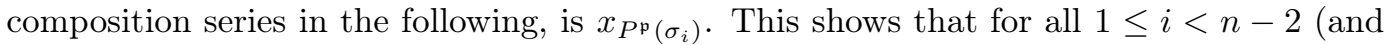
mutatis mutandis also for $i=n-1)$, the composition pairing

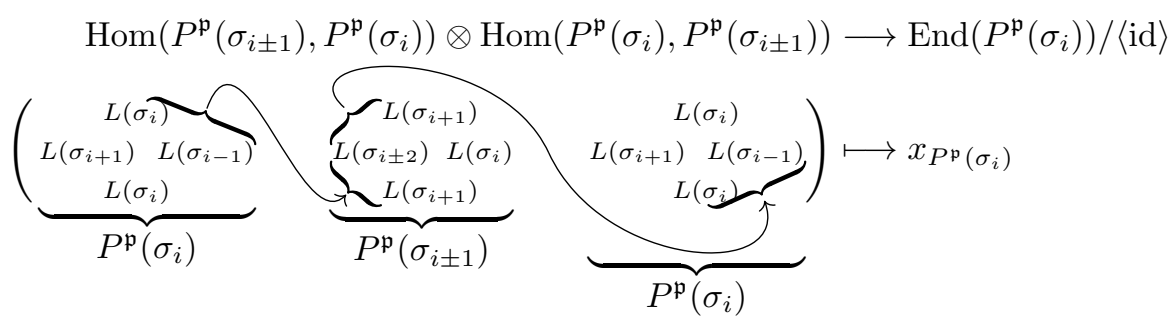

is non-degenerate. 
Theorem 4.14. For the parabolic subalgebra $\mathfrak{p} \subseteq \mathfrak{s l}_{n}$ corresponding to the subgroup $W_{\mathfrak{p}}=$ $S_{n-1} \times S_{1}<S_{n}$, the auto-equivalences $\mathbf{L} \mathrm{Sh}_{s_{i}}[1]$ and $T_{P^{\mathfrak{p}}\left(\sigma_{i}\right)}^{\prime}$ of $D^{\mathrm{b}}\left(\mathcal{O}_{0}^{\mathfrak{p}}\right)$ are naturally isomorphic for every $1 \leq i \leq n-1$.

Proof. Let $A_{n}^{\mathfrak{p}}$ and $Q_{n}^{\mathfrak{p}}$ respectively be the path algebra quotient and the quiver from Lemma 4.12 for $\mathfrak{s l}_{n}$. The assignment $p: A_{n}^{\mathfrak{p}} \rightarrow A_{n}^{\mathfrak{p}} /\left(\varepsilon_{\sigma_{0}}\right) \stackrel{\cong}{\rightrightarrows} A_{n-1}^{\mathfrak{p}}$ induces fully faithful functor

$$
p^{*}: \mathcal{O}_{0}^{\mathfrak{p}}\left(\mathfrak{s l}_{n-1}\right) \rightarrow \mathcal{O}_{0}^{\mathfrak{p}}\left(\mathfrak{s l}_{n}\right), \quad P^{\mathfrak{p}}(e) \mapsto M^{\mathfrak{p}}\left(\sigma_{1}\right), \quad P^{\mathfrak{p}}\left(\sigma_{i-1}\right) \mapsto P^{\mathfrak{p}}\left(\sigma_{i}\right) \text { for } 1 \leq k \leq n-2,
$$

which exhibits $\mathcal{O}_{0}^{\mathfrak{p}}\left(\mathfrak{s l}_{n-1}\right)$ as a full subcategory of $\mathcal{O}_{0}^{\mathfrak{p}}\left(\mathfrak{s l}_{n}\right)$; by induction, it follows that on the triangulated category of $D^{\mathrm{b}}\left(\mathfrak{s l}_{n}\right)$ generated by $P^{\mathfrak{p}}\left(\sigma_{2}\right), \ldots, P^{\mathfrak{p}}\left(\sigma_{n-1}\right)$ there are natural isomorphisms $\mathbf{L} \operatorname{Sh}_{s_{i}}[1] \cong T_{P^{\mathfrak{p}}\left(\sigma_{i}\right)}^{\prime}$ for $2 \leq i \leq n-1$. We shall see, however, that it is easier to show the statement also for $i=1$ and all of $D^{\mathrm{b}}\left(\mathfrak{s l}_{n}\right)$ directly, without resorting to induction, employing the proof of proposition 4.11 with the following alterations:

Since

$$
\operatorname{Hom}_{\mathcal{O}}\left(P^{\mathfrak{p}}\left(\sigma_{i}\right), P^{\mathfrak{p}}\left(\sigma_{j}\right)\right)=0 \quad \text { for } 0 \leq i, j \leq n-1 \text { with }|i-j| \geq 2
$$

it follows from the definition of $T^{\prime}$ that $T_{P^{\mathfrak{p}}\left(\sigma_{i}\right)}^{\prime}$ (for $1 \leq i \leq n-1$ ) acts as identity on all $P^{\mathfrak{p}}\left(\sigma_{j}\right)$ for $0 \leq j \leq n-1$ with $|i-j| \geq 2$, as does, according to table 4.4 , the functor $\mathbf{L} \operatorname{Sh}_{s_{i}}[1]$. One checks that both functors act by identity also on morphisms between these modules; this shows that $T_{P\left(\sigma_{i}\right)}^{\prime} \cong \mathbf{L S h}_{s_{i}}[1]$ for all $1 \leq i \leq n-1$ as functors on the triangulated subcategory of $D^{\mathrm{b}}\left(\mathcal{O}_{0}^{\mathfrak{p}}\left(\mathfrak{s l}_{n}\right)\right)$ generated by these $P^{\mathfrak{p}}\left(\sigma_{j}\right)$ 's.

The category $\mathcal{O}\left(\mathfrak{s l}_{n}\right)$ has a projective generator $P_{n}^{\mathfrak{p}}:=\bigoplus_{k=0}^{n-1} P^{\mathfrak{p}}\left(\sigma_{k}\right)$, with image $\Theta_{s_{i}} P_{n}^{\mathfrak{p}}=$ $P^{\mathfrak{p}}\left(\sigma_{i}\right)^{4}$ for all $1 \leq i \leq n-1$. Again due to table 4.4 , we obtain that

$$
M_{\Theta_{s_{i}}}=\operatorname{Hom}_{\mathcal{O}_{0}}\left(P_{n}^{\mathfrak{p}}, \Theta_{s_{i}} P_{n}^{\mathfrak{p}}\right)=\operatorname{Hom}_{\mathcal{O}_{0}}\left(P_{n}^{\mathfrak{p}}\left(\sigma_{i}-1\right) \oplus P_{n}^{\mathfrak{p}}\left(\sigma_{i}\right) \oplus P_{n}^{\mathfrak{p}}\left(\sigma_{i}+1\right), P_{n}^{\mathfrak{p}}\left(\sigma_{i}\right)^{4}\right)
$$

Replacing $e$ by $\sigma_{i-1}, s$ by $\sigma_{i}$ and $s t$ by $\sigma_{i+1}$ in the proof of proposition 4.11 then gives a proof of $T_{P\left(\sigma_{i}\right)}^{\prime} \cong \mathbf{L} \operatorname{Sh}_{s_{i}}[1]$ for all $1 \leq i \leq n-1$ as functors on $D^{\mathrm{b}}\left(\mathcal{O}_{0}^{\mathfrak{p}}\left(\mathfrak{s l}_{n}\right)\right)$.

\section{Further observations and final remarks}

5.1 $\mathcal{O}_{0}^{\mathfrak{p}}$ as a spherical subcategory We know that the object $P^{\mathfrak{p}}(s) \in \mathcal{O}_{0}^{\mathfrak{p}}\left(\mathfrak{s l}_{3}\right)$ is spherical, so one might ask whether $\mathcal{O}_{0}^{\mathfrak{p}}\left(\mathfrak{s l}_{3}\right)$ arises as the spherical subcategory $\mathcal{S p h}\left(P^{\mathfrak{p}}(s)\right)$ of $P^{\mathfrak{p}}(s) \in$ $\mathcal{O}_{0, \mathfrak{s l}_{3}}$. However, $P^{\mathfrak{p}}(s)$ is not spherelike in $D^{\mathrm{b}}\left(\mathcal{O}\left(\mathfrak{s l}_{3}\right)\right)$, i. e., we cannot assign a meaningful spherical subcategory to it.

To see this, consider the projective resolution $P^{\mathfrak{p}}(s) \simeq\{P(s) \rightarrow P(t s) \rightarrow P(s)\}$ in $D^{\mathrm{b}}\left(\mathcal{O}\left(\mathfrak{s l}_{3}\right)\right)$. Using this resolution, we obtain the chain complex (see section 3.4 for an explanation of the notation)

$$
\begin{aligned}
& \operatorname{hom}_{D^{\mathrm{b}}\left(\mathcal{O}_{0}\right)}^{\bullet}\left(P^{\mathfrak{p}}(s), P^{\mathfrak{p}}(s)\right)
\end{aligned}
$$

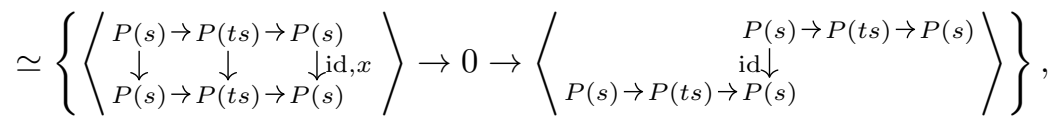

whose leftmost bracket is in degree zero. The complex $\operatorname{hom}_{D^{\mathrm{b}}\left(\mathcal{O}_{0}\right)}\left(P^{\mathfrak{p}}(s), P^{\mathfrak{p}}(s)\right)$ thus has total dimension 3 , so $P^{\mathfrak{p}}(s)$ is not spherelike. As a side note, we notice that the inclusion $D^{\mathrm{b}}\left(\mathcal{O}_{0}^{\mathfrak{p}}\right) \subset D^{\mathrm{b}}\left(\mathcal{O}_{0}\right)$, given by mapping projectives to projectives, is not full. 
5.2 Necessity of extremal partitions Is it necessary to choose a parabolic subalgebra $\mathfrak{p}$ which corresponds to the "extremal" partition $(n-1,1)$, i. e. to the parabolic subgroup $S_{n-1} \times S_{1}<S_{n}$ ? The maximal parabolic subalgebra

$$
\mathfrak{p}=\left(\begin{array}{llll}
* & * & * & * \\
* & * & * & * \\
0 & 0 & * & * \\
0 & 0 & * & *
\end{array}\right) \subset \mathfrak{s l}_{4},
$$

corresponding to the parabolic subgroup $W_{\mathfrak{p}}=\langle s\rangle \times\langle u\rangle=S_{2} \times S_{2}<S_{4}=\langle s, t, u\rangle$ with minimal length coset representatives $W^{\mathfrak{p}}=\{e, t, t u, t s, t s u, t s u t\}$ has parabolic Verma modules and indecomposable projectives with composition series listed in table 5.1; these can be obtained from parabolic Kazhdan-Lusztig polynomials (see also [BS11b]). One checks from the composition series that $P^{\mathfrak{p}}(t), P^{\mathfrak{p}}(t s)$ and $P^{\mathfrak{p}}(t s u)$ are the only spherelike indecomposable projectives, and there no possible $\mathrm{A}_{3}$ configuration of indecomposable projectives. One might wonder if one can find at least an $\mathrm{A}_{2}$-configuration or a single spherical object.

Unfortunately, we encounter the same problem of the present spherelike objects as in the non-parabolic $\mathfrak{s l}_{3}$-case; this can be seen from the composition series as follows. The projectives $P^{\mathfrak{p}}(t s), P^{\mathfrak{p}}(t u)$ corresponding to the two incomparable weights $t s \notin t u$ have non-trivial morphisms $P^{\mathfrak{p}}(t s) \rightarrow P^{\mathfrak{p}}(t u)$ and $P^{\mathfrak{p}}(t u) \rightarrow P^{\mathfrak{p}}(t s)$ whose composition

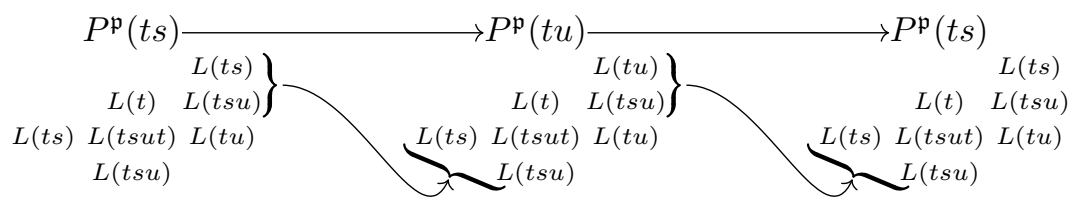

is the zero morphism. The same holds true for $\left(P^{\mathfrak{p}}(t u) \rightarrow P^{\mathfrak{p}}(t) \rightarrow P^{\mathfrak{p}}(t u)\right)=0$. Hence neither $P^{\mathfrak{p}}(t u)$ nor $P^{\mathfrak{p}}(t s)$ is spherical. For $P^{\mathfrak{p}}(t)$ the composition

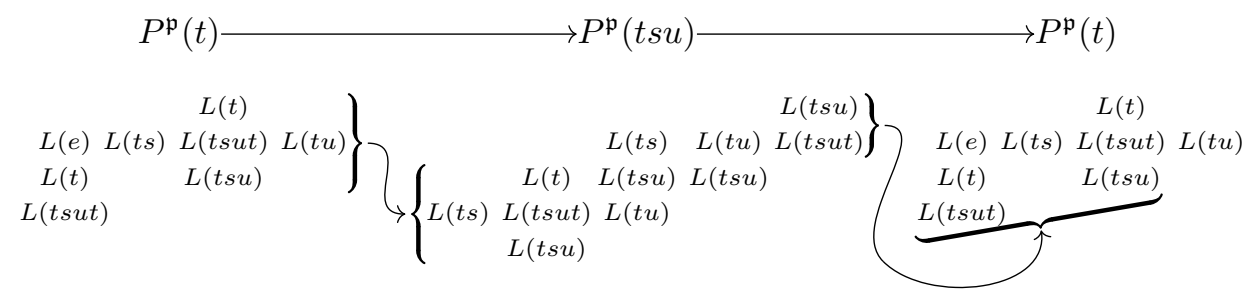

shows that $P^{\mathfrak{p}}(t)$ it is not spherical either.

Therefore, for $\mathfrak{p} \subseteq \mathfrak{s l}_{4}$ the parabolic subalgebra corresponding to the parabolic subgroup $S_{2} \times S_{2} \leq S_{4}$, the modules $P^{\mathfrak{p}}(t), P^{\mathfrak{p}}(t s)$ and $P^{\mathfrak{p}}(t u)$ are the only spherelike indecomposable projective modules, and none of them is spherical.

Acknowledgements This article compiles the results of a Master's thesis written with the advice of Catharina Stroppel, whom the author wants to thank for her continuous support as well as the opportunity provided to dive into this topic.

\section{References}

[ARS95] M. Auslander, I. Reiten and S. O. Smalø. Representation Theory of Artin Algebras. Cambridge Studies in Advanced Mathematics 36. Cambridge, UK: Cambridge University Press, 1995. ISBN: 9780511623608 . DOI: 10.1017/CB09780511623608. 
Table 5.1: Composition series of parabolic Verma modules and indecomposable projectives in $\mathcal{O}_{0}^{\mathfrak{p}}$ for $\mathfrak{p} \subseteq \mathfrak{s l}_{4}$ the parabolic subalgebra corresponding to the parabolic subgroup $W_{\mathfrak{p}}:=S_{2} \times S_{2} \leq S_{4}$ (see $(5.1))$.

\begin{tabular}{|c|c|c|c|c|c|c|c|}
\hline$w \in W^{\mathfrak{p}}$ & $M^{\mathfrak{p}}(w)$ & \multicolumn{6}{|c|}{$P^{\mathfrak{p}}(w)$} \\
\hline$e$ & $\begin{array}{c}L(e) \\
L(t) \\
L(t s u t) \\
\end{array}$ & \multicolumn{6}{|c|}{ dto. } \\
\hline$t$ & $\begin{array}{c}L(t) \\
L(t s) L(t s u t) L(t u) \\
L(t s u)\end{array}$ & & $\begin{array}{r}L(e) \\
L(t) \\
L(t s u t \\
\end{array}$ & 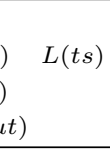 & $\begin{array}{c}L(t) \\
L(t s u t) \\
L(t s u)\end{array}$ & t) $L(t u)$ & \\
\hline ts & $\begin{array}{c}L(t s) \\
L(t s u)\end{array}$ & & $L(t s)$ & $\begin{array}{c}L(t) \\
L(t s u t) \\
L(t s u)\end{array}$ & $L(t s u)$ & $\begin{array}{c}L(t s) \\
L(t s u)\end{array}$ & \\
\hline$t u$ & $\begin{array}{c}L(t u) \\
L(t s u)\end{array}$ & & $L(t s)$ & $\begin{array}{c}L(t) \\
L(t s u t) \\
L(t s u) \\
\end{array}$ & $L(t s u)$ & $\begin{array}{r}L(t u) \\
L(t s u)\end{array}$ & \\
\hline tsu & $\begin{array}{l}L(t s u) \\
L(t s u t)\end{array}$ & $L(t s)$ & $\begin{array}{c}L(t) \\
L(t s u t) \\
L(t s u) \\
\end{array}$ & $L(t s u)^{1}$ & $\begin{array}{c}L(t s) \\
L(t s u)\end{array}$ & $\begin{array}{c}L(t u) \\
L(t s u)\end{array}$ & $\begin{array}{l}L(t s u) \\
L(t s u t)\end{array}$ \\
\hline tsut & $L($ tsut $)$ & $\begin{array}{c} \\
\\
L(e) \\
L(t) \\
L(t s u t\end{array}$ & $L(t s)$ & $\begin{array}{c}L(t) \\
L(t s u t) \\
L(t s u)\end{array}$ & $L(t u)$ & $\begin{array}{l}L(t s u) \\
L(t s u t)\end{array}$ & $L(t s u t)$ \\
\hline
\end{tabular}

[ASS06] I. Assem, D. Simson and A. Skowronski. Elements of the Representation Theory of Associative Algebras. Vol. 1. London Mathematical Society Student Texts 65. Cambridge, UK: Cambridge University Press, 2006. ISBN: 9780511614309. DOI: $10.1017 /$ CB09780511614309.

[Bar15] M. Barot. Introduction to the Representation Theory of Algebras. Springer, 2015. ISBN: 978-3-319-11474-3. DOI: 10.1007/978-3-319-11475-0.

[Bas68] H. Bass. Algebraic K-Theory. 1st ed. New York, Amsterdam: W. A. Benjamin, 1968.

[BB81] A. Beilinson and J. Bernstein. 'Localisation de g-modules'. In: C. R. Acad. Sci. Paris. Série I : Sciences mathémathique 292.1 (1981), pp. 15-18. URL: http://gallica.bnf .fr/ark: /12148/bpt6k6226873r/f32.image.

[Ben95] D. J. Benson. Representations and Cohomology. Vol. 1. Cambridge Studies in Advanced Mathematics 30. Cambridge, UK: Cambridge University Press, 1995. IsBn: 9780521636537.

[BGG76] I. N. Bernstein, I. M. Gelfand and S. I. Gelfand. 'A certain category of $\mathfrak{g}$-modules'. In: Funkcional. Anal. i Prilozen. 10.2 (1976), pp. 1-8. 
[BGS96] A. Beilinson, V. Ginzburg and W. Soergel. 'Koszul duality patterns in representation theory'. In: J. Amer. Math. Soc. 9.2 (1996), pp. 473-527. DOI: 10.1090/S0894-0347-9600192-0.

[BK81] J.-L. Brylinski and M. Kashiwara. 'Kazhdan-Lusztig conjecture and holonomic systems'. In: Invent. Math. 64.3 (1981), pp. 387-410. DOI: 10.1007/BF01389272.

[BS11a] J. Brundan and C. Stroppel. 'Highest Weight Categories Arising from Khovanov's Diagram Algebra I: Cellularity'. In: Mosc. Math. J. 11.4 (2011), pp. 685-722, 821-822. arXiv: 0806.1532.

[BS11b] J. Brundan and C. Stroppel. 'Highest Weight Categories Arising from Khovanov's Diagram Algebra III: Category $\mathcal{O}^{\prime}$. In: Represent. Theory 15 (2011), pp. 70-243. DOI: 10.1090/S1088-4165-2011-00389-7.

[Car86] K. J. Carlin. 'Extensions of Verma modules'. In: Trans. Amer. Math. Soc. 294.1 (1986), pp. 29-43. DOI: $10.2307 / 2000116$.

[CC87] D. H. Collingwood and L. G. Casian. 'The Kazhdan-Lusztig Conjecture for Generalized Verma Modules'. In: Math. Z. 195 (1987), pp. 581-600. DOI: 10 . 1007 /bf01166705. EUDML: 183714 .

[Deo87] V. Deodhar. 'On some geometric aspects of Bruhat orderings II. The parabolic analogue of Kazhdan-Lusztig polynomials'. In: J. Algebra 111.2 (1987), pp. 483-506. DOI: 10.1016/ 0021-8693(87)90232-8.

[Gab80] P. Gabriel. 'Auslander-Reiten sequences and representation-finite algebras'. In: Representation theory. Workshop on the Present Trends in Representation Theory (Ottawa, 1979). Ed. by V. Dlab and P. Gabriel. Vol. 1. Lecture Notes in Mathematics 831. Berlin: Springer, 1980, pp. 1-71. ISBN: 3540102639.

[Gro16] M. Groth. 'Book Project on Derivators'. 2016. URL: http://www. math.uni-bonn.de/ mgroth/monos/intro-to-der-1.pdf.

[Gro57] A. Grothendieck. 'Sur quelques points d'algèbre homologique'. In: Tôhoku Mathematical Journal. 2nd ser. 9 (1957), pp. 119-221. DOI: 10.2748/tmj/1178244839.

[HKP16] A. Hochenegger, M. Kalck and D. Ploog. 'Spherical subcategories in algebraic geometry'. In: Math. Nachr. 289.11-12 (2016), pp. 1450-1465. DOI: 10.1002/mana.201400232. arXiv: 1208.4046 .

[Hum08] J. E. Humphreys. Representations of Semisimple Lie Algebras in the BGG Category $\mathcal{O}$. Graduate Studies in Mathematics 94. American Mathematical Society, 2008. ISBN: 978-0-8218-4678-0.

[Hum72] J. E. Humphreys. Introduction to Lie Algebras and Representation Theory. Graduate Texts in Mathematics 9. New York: Springer, 1972. ISBN: 978-1-4612-6398-2.

[Hum90] J. E. Humphreys. Reflection groups and Coxeter groups. Cambridge Studies in Advanced Mathematics 29. Cambridge University Press, 1990.

[Irv88] R. S. Irving. 'The socle filtration of a Verma module'. In: Ann. Sci. ENS. 4th ser. 21 (1988), pp. 47-65. DOI: 10.24033/asens. 1550.

[Irv90] R. S. Irving. A filtered category $\mathcal{O}_{S}$ and applications. Vol. 1. 6 vols. Memoirs of the American Mathematical Society 419. Providence, RI: Amer. Math. Soc., 1990. ISBn: 978-0-8218-2482-5.

[Irv93] R. S. Irving. 'Shuffled Verma modules and principal series modules over complex semisimple Lie algebras'. In: J. London Math. Soc. 2nd ser. 48.2 (1993), pp. 263-277. DOI: $10.1112 / \mathrm{jlms} / \mathrm{s} 2-48.2 .263$. 
[Jan79] J. C. Jantzen. Moduln mit einem höchsten Gewicht. Lecture Notes in Mathematics 750. Springer, 1979. ISBN: 978-3-540-34854-2.

[KL79] D. Kazhdan and G. Lusztig. 'Representations of Coxeter Groups and Hecke Algebras'. In: Inv. Math. 53 (1979), pp. 165-184. DOI: 10.1007/bf01390031.

[KM16] T. Kildetoft and V. Mazorchuk. 'Parabolic Projective Functors in Type A'. In: Adv. Math. 301 (2016), pp. 785-803. DOI: 10.1016/j.aim.2016.06.026. arXiv: 1506.07008.

[Kra07] H. Krause. 'Derived categories, resolutions, and Brown representability'. In: Interactions between Homotopy Theory and Algebra. Summer School on the Interactions between Homotopy Theory and Algebra (Chicago, 2004). Contemporary Mathematics 436. Providence, RI: AMS, 2007, pp. 101-145. arXiv: 0511047.

[LS13] T. Lejczyk and C. Stroppel. 'A graphical description of $\left(\mathrm{D}_{n}, \mathrm{~A}_{n-1}\right)$ Kazhdan-Lusztig polynomials'. In: Glasgow Math. J. 55.2 (2013), pp. 313-340. DOI: 10.1017/S0017089512000547.

[Mar06] F. Marko. 'Algebra associated with the principal block of category $\mathcal{O}$ for $\mathfrak{s l}_{3}(\mathbf{C})$ '. In: Algebras, Rings and Their Representations. International Conference on Algebras, Modules and Rings (Lisbon, 14th-18th July 2003). Ed. by A. Facchini et al. 2006, pp. 201-2014. ISBN: 978-981-256-598-3.

[Maz12] V. Mazorchuk. Lectures on Algebraic Categorification. Zurich: Eur. Math. Soc., 2012. ISBN: 978-3-03719-108-8.

[MS05] V. Mazorchuk and C. Stroppel. 'Translation and shuffling of projectively presentable modules and a categorification of a parabolic Hecke module'. In: Trans. Amer. Math. Soc. 357 (2005), pp. 2939-2973. DOI: 10.1090/S0002-9947-04-03650-5.

[MS08] V. Mazorchuk and C. Stroppel. 'Projective-injective modules, Serre functors and symmetric algebras'. In: J. reine angew. Math. 616 (2008), pp. 131-165. DOI: 10.1515/CRELLE. 2008.020.

[Roc80] A. Rocha-Caridi. 'Splitting Criteria for $\mathfrak{g}$-Modules Induced from a Parabolic and the Bernstein-Gelfand-Gelfand Resolution of a Finite Dimensional, Irreducible g-Module'. In: Trans. Amer. Math. Soc. 2 (1980), pp. 335-366. DOI: 10.2307/1999832. JSTOR: 1999832.

[Rou06] R. Rouquier. 'Categorification of $\mathfrak{s l}_{2}$ and braid groups'. In: Trends in representation theory of algebras and related topics. Contemp. Math. 406. Providence, RI: Amer. Math. Soc., 2006, pp. 137-167. DOI: 10.1090/conm/406/07657. arXiv: math/0409593.

[Soe90] W. Soergel. 'Kategorie $\mathcal{O}$, perverse Garben und Moduln über den Koinvarianten zur Weylgruppe'. In: J. Amer. Math. Soc. 3.2 (1990), pp. 421-445. DOI: 10.1090/S08940347-1990-1029692-5.

[Soe97] W. Soergel. 'Kazhdan-Lusztig polynomials and a combinatoric[s] for tilting modules'. In: Represent. Theory 1 (1997), pp. 83-114. DOI: 10.1090/S1088-4165-97-00021-6.

[ST01] P. Seidel and R. Thomas. 'Braid Group Actions on Derived Categories of Coherent Sheaves'. In: Duke Math. Jour. 108 (2001), pp. 37-108. DOI: 10.1215/s0012-7094-0110812-0.

[Str03a] C. Stroppel. 'Category $\mathcal{O}$ : Gradings and translation functors'. In: J. Algebra 268 (2003), pp. 301-326. DOI: 10.1016/S0021-8693(03)00308-9.

[Str03b] C. Stroppel. 'Category $\mathcal{O}$ : Quivers and endomorphism rings of projectives'. In: Representation Theory 7 (2003). DOI: 10.1090/S1088-4165-03-00152-3.

[Wei94] C. A. Weibel. An introduction to homological algebra. Cambridge studies in advanced mathematics 38. Cambridge: Cambridge University Press, 1994. IsBN: 0-521-43500-5. 